\title{
On geometrically defined extensions of the Temperley-Lieb category in the Brauer category
}

\author{
Z. Kádár ${ }^{1}$. P. P. Martin ${ }^{1} \cdot$ S. Yu ${ }^{2}$
}

Received: 22 January 2015 / Accepted: 11 December 2018 / Published online: 4 March 2019

(c) The Author(s) 2019

\section{Introduction}

The partition algebra and its Brauer and Temperley-Lieb (TL) subalgebras $[10,50,69]$ have many applications and a rich representation theory. See for example [12,34,43,51,59,71]. In particular the representation theory of each of these algebras has an intriguing geometric characterisation $[19,37,48,58]$. The TL case can be understood in terms of Lie Theoretic notions of alcove geometry and geometric linkage [37], via generalised Schur-Weyl duality, and indeed Ringel duality [26,49]. However the Brauer case is much richer, for example in that, as the rank gets large, there are cell modules of arbitrarily large Loewy depth and width [6] even over the complex field [55]; and, although its complex representation theory is now intrinsically well-understood, the paradigm for the corresponding alcove geometric notions is more intriguing. Beautiful links with alcove geometry are implicit in the generalisation to the orthosymplectic case [5] (cf. for example [14,68]) of Brauer's original orthogonal group duality [10]; and appear explicitly in recent work of Ehrig and Stroppel [27-29]. But this is not a straightforward generalisation of the TL case, and the partition algebra case is again different [58].

Here we introduce, for each $l \in\{-1,0,1,2, \ldots\}$, a tower of algebras $J_{l, n}\left(n \in \mathbb{N}_{0}\right)$. Varying $l$ interpolates between the TL algebras, case $l=-1$, and the Brauer algebras, case $l=\infty$. (A general indication of this interpolation is given by comparing the dimensions of the algebras in low rank-see Fig. 1.) A particular aim is to study the geometry in their representation theory by lifting this new connection to the representation theory level. To this end we investigate the representation theory of the new algebras using their amenability to tower of recollement (ToR) [18] and monoid methods [64]. The representation theory for large $l, n$ eventually becomes very hard, but we are able to prove a number of useful general results, and results over the complex field. For example we obtain the 'generic' semisimple structure in the sense of [17].

By way of further motivation (although we will not develop the point here) we note that both the Brauer and TL algebras provide solutions to the Yang-Baxter (YB) equations [4,65], and hence in principle integrable models in statistical mechanics [48]. In addition to their

P. P. Martin

ppmartin@maths.leeds.ac.uk

1 Department of Pure Mathematics, University of Leeds, Leeds, UK

2 Sydney, Australia 
Fig. 1 Table of dimensions of algebras $J_{l, n}$

\begin{tabular}{|r|r|r|r|r|r|r|r|r}
\hline & $n=0$ & 1 & 2 & 3 & 4 & 5 & 6 & \\
\hline$l=-1$ & 1 & 1 & 2 & 5 & 14 & 42 & 132 & \\
\hline 0 & 1 & 1 & 3 & 11 & 43 & 173 & 707 & \\
\hline 1 & 1 & 1 & 3 & 15 & 87 & 525 & & \\
\hline 2 & 1 & 1 & 3 & 15 & 105 & 849 & & \\
\hline 3 & 1 & 1 & 3 & 15 & 105 & 945 & 9795 & \\
\hline$\infty$ & 1 & 1 & 3 & 15 & 105 & 945 & 10395 & \\
\hline
\end{tabular}

interest from a representation theory perspective, our new algebras can be seen as ways to address the problem of construction of natural solutions to the boundary YB equations in the TL setting (generalising the blob approach and so on-see e.g. [23] and references therein). A paradigm here is the XXZ spin chain-a 'toy' model of quantum mechanical interacting spins on a 1-dimensional spacial lattice derived from the Heisenberg model [7]; see e.g. [46, Ch.6] or [14]. The motivation here would be to study spin chains with special physical boundary conditions - conditions penetrating in a way controlled by the parameter $l$ at one end (or both ends) of the chain of length $n$.

An outline of the paper is as follows. The partition category has a basis of set partitions, and the Brauer and Temperley-Lieb categories are subcategories with bases of certain restricted partitions. In particular the Temperley-Lieb category has a basis of non-crossing partitions. (There are two distinct such realisations—-see [48, §6.2.1]. We will mainly focus on the realisation by non-crossing partitions into pairs.) Here we provide a classification of partitions generalising the plane-geometrical notion of non-crossing. Many such games are possible in principle (see e.g. [15]), but we show that our classification (like non-crossing) is preserved under the partition category composition. This closure theorem allows us to define a sequence of new subcategories. Next we turn towards our motivating aim: investigation of the representation theory of algebras contained in these categories. We focus in particular here on the extensions of the Temperley-Lieb category in the Brauer category. In this paper we establish a framework for modular representation theory of the corresponding towers of algebras. In particular we construct for each algebra a set of modules over a suitable integral ground ring that, on base change to the corresponding field of fractions, are a complete set of simple modules. In the case that is modular over $\mathbb{C}$ in the sense of [11] we prove that the algebras are generically semisimple. We observe an intriguing subset of parameter values for which they are not semisimple, distinct from both TL and Brauer cases. We conclude by determining branching rules, and hence give combinatorial constructions for the ranks of these algebras. Finally in Sect. 6 we note some open problems.

The TL algebra has a sequence of known generalisations using its characterisation via an embedding of pair partitions in the plane- the blob algebras and the contour algebras [56]; as well as various beautiful generalisations due to Green et al. [30,33], tom Dieck [70] and others. The blob algebra also has a rich geometrically-characterised representation theory [58]. However none of the previously known cases serve to interpolate between the TL algebra and the Brauer algebra.

\subsection{Preliminary definitions and pictures for set partitions}

We need to recall a pictorial realisation of the partition algebra (i.e. of set partitions). This realisation is in common use (see e.g. [51]), but we will need to develop it more formally.

(1.1) A partition of a set $T$ is a set of subsets of $T$ such that the subsets are pairwise disjoint, and their union is $T$. Let $P(T)$ denote the set of partitions of set $T$. 

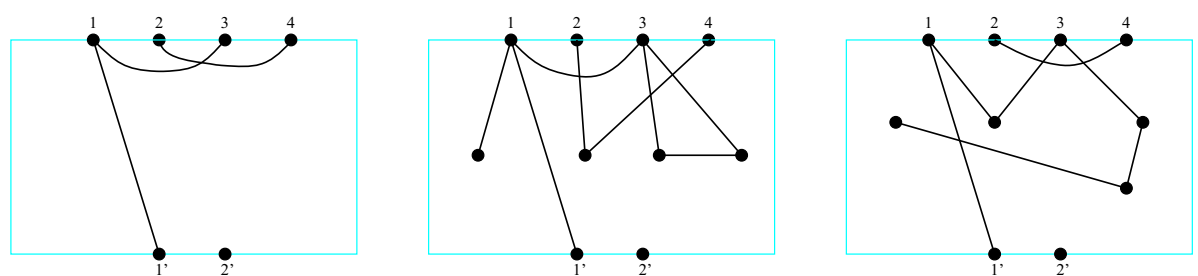

Fig. 2 Graphs for the partition $\left\{\left\{1,3,1^{\prime}\right\},\{2,4\},\left\{2^{\prime}\right\}\right\}$ of the set $\left\{1,2,3,4,1^{\prime}, 2^{\prime}\right\}$

Noting the standard bijection between $P(T)$ and the set of equivalence relations on $T$ we write $a \sim^{p} b$ when $a, b$ in the same part in $p \in P(T)$.

Suppose that $p \in P(T)$ and $S \subset T$. Write $\left.p\right|_{S}$ for the restriction of $p$ to $S$. Write $f_{S}(p):=\#\{\pi \in p \mid \pi \cap S=\emptyset\}$, the number of parts of $p$ that do not intersect $S$. (Here we follow [50, Def. 20]. See also e.g. [53].)

(1.2) Let $\mathcal{G}$ denote the class of graphs; $\mathcal{G}(V)$ the subclass of graphs on finite vertex set $V$; and $\mathcal{G}[S]$ the subclass of $\mathcal{G}$ of graphs whose vertex set contains set $S$. Define

$$
\Pi: \mathcal{G}(V) \rightarrow P(V)
$$

by $v \sim \Pi(g) v^{\prime}$ if $v, v^{\prime}$ are in the same connected component in the graph $g$. Define

$$
\Pi_{S}: \mathcal{G}[S] \rightarrow P(S)
$$

by $\Pi_{S}(g)=\left.\Pi(g)\right|_{S}$. Note that additional edges between vertices joined by an edge in $g$ have no effect on $\Pi_{S}(g)$.

(1.3) We shall use drawings to represent graphs in a conventional way: vertices by points and edges by polygonal arcs between vertex points, as in Fig. 2 or Fig. 3. We shall refer to these polygonal arcs as lines for brevity.

A picture $d$ of a graph is thus (i) a rectangular region $R$ of the plane; (ii) an injective map from a finite set into $R$ (hence a finite subset of points identified with vertices); and (iii) a subset of $R$ that is the union of lines. Line crossings are not generally avoidable (in representing a given graph in this way), but we stipulate 'line regularity': that, endpoints apart, lines touch only at points in the interior of straight segments; and that a line does not touch any vertex point except its endpoints, or the boundary of $R$ except possibly at its endpoints.

Note: (I) Any finite graph can be represented this way (indeed with the vertices in any position, see e.g. [20]). (II) The regularity condition ensures that a picture gives the path of each line unambiguously. Thus no two distinct graphs have the same picture, and indeed the process of following the path of each line gives us a map 'back' from pictures to graphs.

For $g \in \mathcal{G}[S]$ one thinks of $S$ as a set of 'external' vertices, and draws them on the horizontal part of the rectangle boundary. Interior vertices $(v \notin S)$ will generally not need to be explicitly labelled here (the choice of label will be unimportant).

By (I) and (II), via $\Pi_{S}$, we can use a picture of $g \in \mathcal{G}[S]$ to represent a partition. The drawings in Fig. 2 all represent the same partition, when regarded as pictures of set partitions of $S=\left\{1,2,3,4,1^{\prime}, 2^{\prime}\right\}$. Specifically in each case $\Pi_{S}(g)=\left\{\left\{1,3,1^{\prime}\right\},\{2,4\},\left\{2^{\prime}\right\}\right\}$.

(1.4) A vacuum bubble in $g \in \mathcal{G}[S]$ is a purely interior connected component [9] (cf. Fig. 3). The vacuum bubble number is

$$
\Pi_{S}^{f}(g)=f_{S}(\Pi(g))=\#\{\pi \in \Pi(g) \mid \pi \cap S=\emptyset\} .
$$



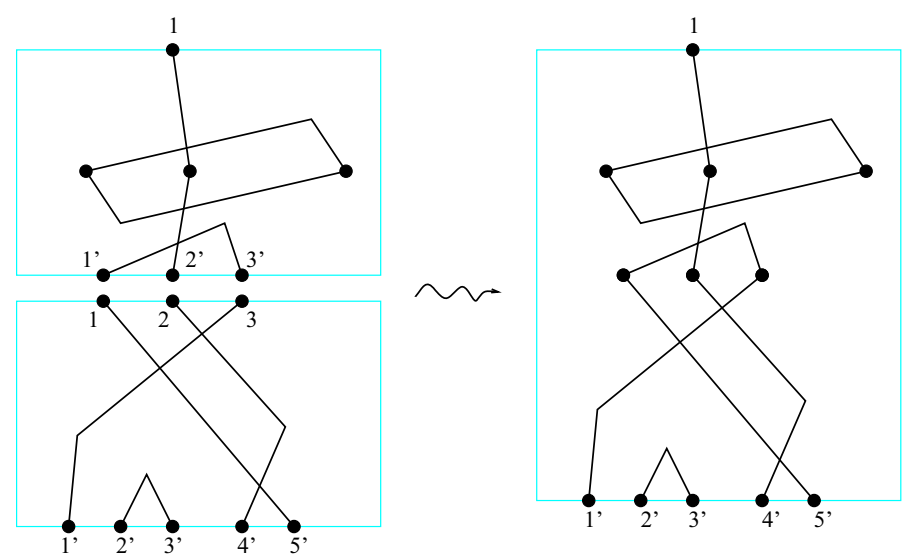

Fig. 3 Picture stacking composition

(1.5) Let $\underline{n}:=\{1,2, \ldots, n\}$ and $\underline{n}^{\prime}:=\left\{1^{\prime}, 2^{\prime}, \ldots, n^{\prime}\right\}$, and so on. Let

$$
P(n, m):=P\left(\underline{n} \cup \underline{m}^{\prime}\right) .
$$

(1.6) An $(n, m)$-graph is an element of $\mathcal{G}(n, m):=\mathcal{G}\left[\underline{n} \cup \underline{m}^{\prime}\right]$. We draw them as in Figs. 2 and 3. We define $\Pi_{n, m}=\Pi_{\underline{n} \cup \underline{m}^{\prime}}$, so

$$
\Pi_{n, m}: \mathcal{G}(n, m) \rightarrow P(n, m) .
$$

(1.7) Next we recall the partition category $\mathcal{P}$, as defined in [50, §7]. We first fix a commutative ring $k$ say, and $\delta \in k$. The object set in $\mathcal{P}$ is $\mathbb{N}_{0}$. The arrow set $\mathcal{P}(n, m)$ is the free $k$-module with basis $P(n, m)$. Noting (1) this means that elements of $\mathcal{P}(n, m)$ could be represented as formal $k$-linear combinations of $(n, m)$-graphs. In fact one generalises this slightly. In $\mathcal{P}$ an $(n, m)$-graph [as in (1.6)] maps to an element of $k P(n, m)$ via:

$$
\Pi_{\mathcal{P}}: g \mapsto \delta^{\Pi_{n, m}^{f}(g)} \Pi_{n, m}(g) .
$$

For example the upper picture on the left in Fig. 3 encodes $\delta^{1}\left\{\left\{1,2^{\prime}\right\},\left\{1^{\prime}, 3^{\prime}\right\}\right\}$.

The composition $p * q$ in $\mathcal{P}$ can be defined and computed in naive set theory [50]. But it can also be computed by representing composed partitions as stacks of corresponding pictures of graphs, as in Fig. 3. First a composition $\circ: \mathcal{G}(n, m) \times \mathcal{G}(m, l) \rightarrow \mathcal{G}(n, l)$ is defined: $a \circ b$ is the combination of graphs with $m$ vertex subsets identified and de-labelled as indicated in Fig. 3. Here we only use the pictures as a convenience to indicate the vertex assignments in the new graph. However by stacking pictures of $a$ and $b$ so that the $m$ vertex sets in each meet and are identified as in the figure, we do get a picture of $a \circ b$. Then

$$
p * q=\Pi_{\mathcal{P}}(a \circ b)
$$

for suitable $a, b$. For example, in case $p=\left\{\left\{1,2^{\prime}\right\},\left\{1^{\prime}, 3^{\prime}\right\}\right\}$ in $P(1,3)$ and $q=$ $\left\{\left\{1,5^{\prime}\right\},\left\{2,4^{\prime}\right\},\left\{3,1^{\prime}\right\},\left\{2^{\prime}, 3^{\prime}\right\}\right\}$ in $P(3,5)$ then the composition $\delta p * q$, or more explicitly

$$
\delta\left\{\left\{1,2^{\prime}\right\},\left\{1^{\prime}, 3^{\prime}\right\}\right\} *\left\{\left\{1,5^{\prime}\right\},\left\{2,4^{\prime}\right\},\left\{3,1^{\prime}\right\},\left\{2^{\prime}, 3^{\prime}\right\}\right\}=\delta\left\{\left\{1,4^{\prime}\right\},\left\{1^{\prime}, 5^{\prime}\right\},\left\{2^{\prime}, 3^{\prime}\right\}\right\}
$$

can be verified via Fig. 3. The independence of $p * q$ on the choice of $a, b$ such that $\Pi_{\mathcal{P}}(a)=p$ and $\Pi_{\mathcal{P}}(b)=q$ follows since connected components in $a \circ b$ are chains of paths in $a$ and $b$, 
but any $a^{\prime} \in \Pi_{\mathcal{P}}^{-1}(p)$ has a path between two vertices if and only if $a$ has one. Denoting the stack of pictures for $a \circ b$ by $d \mid d^{\prime}$, top to bottom, then we can write

$$
p * q=\Pi_{\mathcal{P}}\left(d \mid d^{\prime}\right)
$$

for any $d, d^{\prime}$ such that $p=\Pi_{\mathcal{P}}(d)$ and $q=\Pi_{\mathcal{P}}\left(d^{\prime}\right)$. To check associativity of $*$ note that $a *(b * c)$ becomes a stack identical to that for $(a * b) * c$. We have defined the category

$$
\mathcal{P}=\left(\mathbb{N}_{0}, k P(n, m), *\right) .
$$

This is equivalent to the original set theoretic definition [50].

Remark From this partition category perspective the pictures constitute a mild modification of the plane projection of arrows in the tangle category, in which arrows ('morphisms') are certain collections of non-intersecting polygonal arcs in a 3D box (see later, or e.g. [39]).

Write $J(n, m) \subset P(n, m)$ for the subset of partitions of $\underline{n} \cup \underline{m^{\prime}}$ into pairs; and

$$
\mathcal{B}=\left(\mathbb{N}_{0}, k J(n, m), *\right)
$$

for the corresponding Brauer subcategory of $\mathcal{P}$ (see e.g. [53, §5.1] or [44]).

\subsection{Overview of the paper}

We start with a heuristic overview and summary. Later, in order to prove the main Theorems, we will give more formal definitions.

Besides the representation of a set partition $p$ by a graph $g$, the task of constructing a picture $d$ of $p$ contains another layer-the embedding and depiction of the graph $g$ in the plane. Both stages of the representation of set partitions are highly non-unique. However, they lead to some remarkable and useful invariants. To describe these invariants we will need a little preparation.

Suppose we have a picture $d$ of a partition of this kind. Then each polygonal arc $l$ of $d$ partitions the rectangle $R$ into various parts: one or more connected components of $R \backslash l$; and $l$ itself. Overall, a picture $d$ subdivides $R$ into a number of connected components, called alcoves, of $R \backslash d$ (regarding $d$ as the union of its polygonal arcs), together with $d$ itself.

(1.8) Given a picture $d$, the distance $\mathrm{d}_{d}(x, y)$ from point $x$ to $y$ is the minimum number of polygonal arc segments crossed by a continuous path from $x$ to $y$, among all such paths. Examples (the second picture shows distances to $y$ from points in various alcoves):
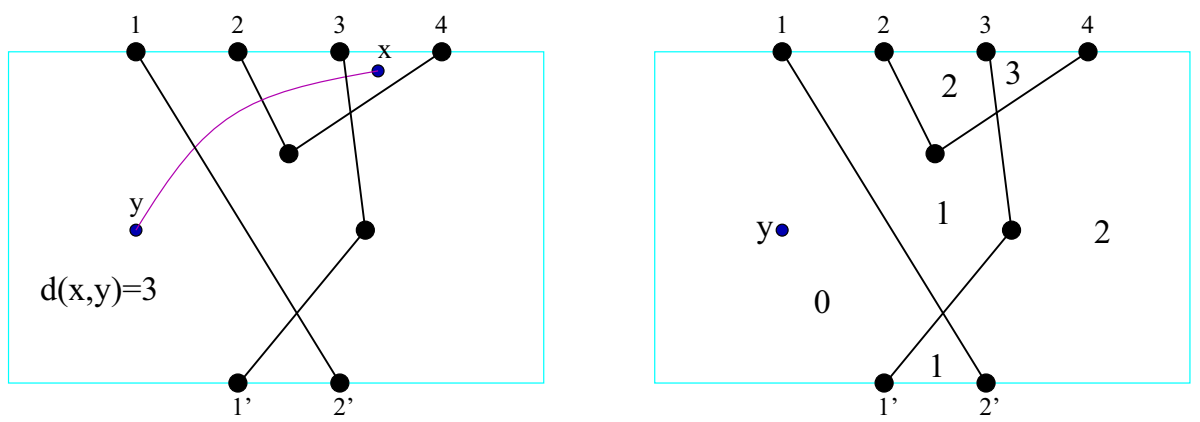

Note that there is a well-defined distance between a point and an alcove; or between alcoves. 
(1.9) The height, or left-height, of a point in $d$ is defined to be the distance from the leftmost alcove. (By symmetry there is a corresponding notion of rightmost alcove; and right-height may then be defined similarly to (left-)height.)

Given a picture $d$, a crossing point is a point where two polygonal arc segments cross. Note that these points in particular have heights. For example the upper of the two crossing points in the picture above has height 1 , and the other has height 0 .

The height ht $(d)$ of a picture $d$ with crossing points is defined to be the maximum height among the heights of its crossing points. We shall say that the height of a picture without crossings is -1 .

(1.10) Although the picture $d$ of a partition $p$ is non-unique, we can ask, for example, if it is possible to draw $p$ without arc crossings-i.e. if among the drawings $d$ of $p$ there is one without crossings. If it is not possible to draw $p$ without crossings, we can ask what is the minimum height needed - that is, among all the pictures $d$ representing $p$, what is the minimum picture height? We call this minimum the height of partition $p$.

(1.11) Returning to the partition category $\mathcal{P}=\left(\mathbb{N}_{0}, k P(n, m), *\right)$, the existence of a Temperley-Lieb subcategory in $\mathcal{P}$ (see e.g. [48, §6.2], [53, §5.1]) corresponds to the observation that the product $p * p^{\prime}$ in $\mathcal{P}$ of two partitions of height -1 (i.e. non-crossing) gives rise to a partition that is again height -1 . Our first main observation is a generalisation of this:

The height of $p * p^{\prime}$ in $\mathcal{P}$ does not exceed the maximum of the heights of $p, p^{\prime}$. Thus:

For each $l \in \mathbb{N}_{-}:=\{-1,0,1,2, \ldots\}$ there is a subcategory spanned by the partitions of height at most $l$.

We first prove this result. This requires formal definitions of 'left-height' and so on, and then some mildly geometrical arguments. Recall that $J(n, m) \subset P(n, m)$ is the subset of partitions of $\underline{n} \cup \underline{m}^{\prime}$ into pairs; and that the partitions of this form span the Brauer subcategory: $\mathcal{B}=\left(\mathbb{N}_{0}, k \bar{J}(n, m), *\right)$. The construction above induces a sequence of subcategories here too. The rest of the paper is concerned with the representation theory of the tower of algebras associated to each of these categories, that is, the algebras that are the End-sets in each of these categories.

\section{Formal definitions and notations}

We start with a formal definition of a picture, a drawing as in (1.3). Notation is taken largely from Moise [60] and Crowell-Fox [20].

(2.1) Given a manifold $M$ we write $\partial M$ for the manifold-theoretic boundary; and $(M)=$ $M \backslash \partial M$ for the interior [60, §0].

(2.2) A polygonal arc is an embedding $l$ of $[0,1]$ in $\mathbb{R}^{3}$ consisting of finitely many straightline segments. The open arc $(l)$ of $l$ is the corresponding embedding of $(0,1)$. An arc-vertex in $l$ is the meeting point of two maximal straight segments.

(2.3) A polygonal graph is (i) an embedding $\epsilon$ of the vertex set $V$ of some $g \in \mathcal{G}(V)$ as points in $\mathbb{R}^{3}$; and (ii) for graph edges $E$ a polygonal embedding $\epsilon: \sqcup_{e \in E}(0,1) \hookrightarrow \mathbb{R}^{3} \backslash \epsilon(V)$ such that the closure points of $(0,1)_{e}$ agree with the endpoints of $e$.

(2.4) Note that every $g$ has an embedding for every choice of $\epsilon: V \hookrightarrow \mathbb{R}^{3}$.

(2.5) In our construction the vertex labels in a graph $g$ are important data, but edge labels are unimportant. Note that if the edge labels are unimportant then we can recover the original 
graph from the map $\epsilon: V$ (labeling graph vertex points) and the image $\epsilon(g)$. Note well the distinction between graph vertices $\epsilon(v \in V)$ and polygonal arc-vertices.

(2.6) Fix a coordinate system on $\mathbb{R}^{3}$. A polygonal graph $G=\epsilon(g)$ is regular (in the rectangle $R \subset \mathbb{R}^{2}$ ) if

(i) the projection $p(x, y, z)=(x, y)$ is injective on vertices;

(ii) for $k \in G \backslash \epsilon(V)$ then $\left|p^{-1}(p(k))\right| \leq 2$;

(iii) $\left|p^{-1}(p(k))\right|=1$ if $k$ an arc-vertex;

(iv) for $k \in G, p(k) \in p(\epsilon(V))$ implies $k \in \epsilon(V)$;

(v) $p(G) \subset R$ and $p(k) \in \partial R$ implies $k \in \epsilon(V)$.

(2.7) A picture is a triple $d=(V, \lambda, L)$ consisting of a set $V$, an injective map $\lambda: V \hookrightarrow \mathbb{R}^{2}$ and a subset $L \subset \mathbb{R}^{2}$ such that $\lambda=\left.p \circ \epsilon\right|_{V}$ for some regular polygonal graph with $g \in \mathcal{G}(V)$; and $L$ is the image $L=p(\epsilon(g))$. (The datum also includes the containing rectangle $R$, but notationally we leave this implicit.)

The point here is that such a $d$, consisting only of labeled points and a subset of $R$, determines a graph $g$; and every graph has a picture. Note that $d$ also determines the set of points where $\left|p^{-1}(p(k))\right|=2$ in (2.6)(ii)—the set $\chi(d)$ of crossing points.

(2.8) Let us consider pictures with $R$ oriented so that its edges lie in the $x$ and $y$ directions. If the vertex points on the northern (respectively southern) edge of $R$ are not labelled explicitly then they may be understood to be labeled $1,2, \ldots$ (respectively $1^{\prime}, 2^{\prime}, \ldots$ ) in the natural order from left to right.

In particular such a frame-drawn picture without any vertex point labels determines a graph in some $\mathcal{G}\left[\underline{n} \cup \underline{m}^{\prime}\right]$ up to labelling of the other 'interior' vertices.

We identify pictures differing only by an overall vertical shift. Given our vertex labelling convention above we could also identify under horizontal shifts, but the horizontal coordinate will be a useful tool in proofs later, so we keep it for now.

\subsection{Stack composition of pictures}

Here we follow the usual construction of 'diagram categories' (e.g. as in [50, §7]), but take care to emphasise specific geometrical features that we will need later.

(2.9) Given $d=(V, \lambda, L)$ in rectangle $R$ write $\mathrm{n}(d)$ for the subset of $\mathbb{R}$ giving the intersection of $L$ with the northern edge of $R$ (thus by (2.6)(v) the collection of $x$-values of 'northern' exterior vertex points, or 'marked points'). Write $\mathrm{s}(d)$ for the corresponding southern set.

Write $\mathrm{h}_{0}(a, b)$ for the class of pictures $d$ with $\mathrm{n}(d)=a$ and $\mathrm{s}(d)=b$, and $L$ not intersecting the two vertical edges of the containing rectangle.

(2.10) Note that for $d \in \mathrm{h}_{0}(a, b)$ there is an essentially identical picture with $R$ wider. Thus any two such pictures may be taken to have the same (unspecified, finite) interval of $\mathbb{R}$ as their northern edges, and southern edges. The juxtaposition of rectangles, $R, R^{\prime}$ say, by vertical stacking of $R$ over $R^{\prime}$ thus produces a rectangle, denoted $R \mid R^{\prime}$. This is almost a disjoint union, except that the southern edge of $R$ is identified with the northern edge of $R^{\prime}$.

Given a pair of pictures $d$ and $d^{\prime}$, stack $R \mid R^{\prime}$ induces a corresponding pair of subsets $\lambda(V) \mid \lambda^{\prime}\left(V^{\prime}\right)$ and $L \mid L^{\prime}$ in the obvious way. For example see Fig. 3.

Proposition 2.11 The stack juxtaposition of a picture $d$ in $\mathrm{h}_{0}(a, b)$ over a picture $d^{\prime}$ in $\mathrm{h}_{0}(b, c)$ defines a picture $d \mid d^{\prime}$ in $\mathrm{h}_{0}(a, c)$. 
Proof As noted, the stack $R \mid R^{\prime}$ induces a corresponding pair of subsets $\lambda(V) \mid \lambda^{\prime}\left(V^{\prime}\right)$ and $L \mid L^{\prime}$. The former is a union of finite point sets which clearly agrees with $a$ and $c$ on the relevant edges of $R \mid R^{\prime}$. The latter is a union of lines, and the only new meetings are at the marked points in $b$ (as it were). These are now interior marked points. Conditions (2.6)(i-v) hold by construction.

Remark 2.12 Recall from (2.8) that we identify pictures differing by overall vertical shift, but not up to isotopy here. Note also that for $d \in \mathrm{h}_{0}(a, a)$ with $a$ non-empty then $d$ has at least $2|a|$ vertices (the northern and southern exterior vertices), unless we allow rectangles of zero vertical extent. Consider $d, d^{\prime} \in \mathrm{h}_{0}(a, a)$. If $d$ has $v$ vertices and $d^{\prime}$ has $v^{\prime}$ vertices then $d \mid d^{\prime}$ has $v+v^{\prime}-|a|$ vertices. Thus stack composition of pictures of non-zero vertical extent always produces a picture with more (internal) vertices than either. Thus no such picture can be an identity of stack composition. On the other hand, allowing rectangles of zero vertical extent in any $\mathrm{h}_{0}(a, a)$ allows for an identity element $1_{a}$ of stack composition in $\mathrm{h}_{0}(a, a)$. Write $h_{0}$ for the 'picture category'.

Proposition 2.13 For finite subsets $a$ and $b$ of $\mathbb{R}$, there is a surjection

$$
\pi_{e}: \mathrm{h}_{0}(a, b) \rightarrow P(|a|,|b|)
$$

given by counting the elements of a (resp. b) from left to right and hence passing to a graph by (2.8), and then using $\Pi_{n, m}$ from (1).

Proposition 2.14 Fix a commutative ring $k$ and $\delta \in k$, and let $\pi_{p}$ denote the generalisation of $\pi_{e}$ corresponding to $\Pi_{\mathcal{P}}$ from (1.7). Let $d \in \mathrm{h}_{0}(a, b), d^{\prime} \in \mathrm{h}_{0}(b, c)$. Then $\pi_{p}\left(d \mid d^{\prime}\right)=\pi_{p}(d) * \pi_{p}\left(d^{\prime}\right)$, where the product on the right is in the partition category $\mathcal{P}=\left(\mathbb{N}_{0}, k P(n, m), *\right)$ as in $(1.7)$.

Proof Note from (2.7) and (2.8) that we have a well-defined map from pictures to graphs, and hence in particular maps $\kappa: \mathrm{h}_{0}(a, b) \rightarrow \mathcal{G}(|a|,|b|)$. In (1.7) $\mathcal{P}$ is defined using these graph sets $\mathcal{G}(n, m)$ and the map $\Pi_{\mathcal{P}}$. By construction we have $\pi_{p}=\Pi_{\mathcal{P}} \circ \kappa$, that is, $\pi_{p}$ factors through $\mathcal{G}(n, m)$.

(2.15) If $d \in \mathrm{h}_{0}(a, b)$ is a picture as above, let $d^{*} \in \mathrm{h}_{0}(b, a)$ denote the picture obtained by flipping $d$ top-to-bottom.

Let $p \in P(n, m)$. Write $p^{*}$ for the element of $P(m, n)$ obtained by swapping primed and unprimed elements of the underlying set.

Note that if $d$ is a picture of $p \in P(n, m)$ then $d^{*}$ is a picture of $p^{*} \in P(m, n)$. Furthermore, this $*$ is a contravariant functor between the corresponding partition categories.

(2.16) Note that for any picture in the category $h_{0}$ with distinct northern and southern edge we can vertically rescale to arbitrary finite separation of these edges. Thus we can make any two pictures have the same separation. For two such pictures $d, d^{\prime}$ there is a picture $d \otimes d^{\prime}$ obtained by side-by-side juxtaposition.

(2.17) We call a picture a chain picture if every exterior marked point [as in (2.9)] is an endpoint of precisely one line, and every interior marked point is an endpoint of precisely two lines (e.g. as in Fig. 3). Write $\mathrm{h}_{0}^{2}(a, b) \subset \mathrm{h}_{0}(a, b)$ for the subset of chain pictures. Note that every $p \in J(n, m)$ has a chain picture. We have the following.

Lemma 2.18 The stack composition Proposition 2.11 closes on the subset of chain pictures (i.e. if $d, d^{\prime}$ are chain pictures then so is $d \mid d^{\prime}$ ). This gives a subcategory of the category in Remark 2.12. The corresponding $\pi_{p}$-quotient category (as in Proposition 2.14) coincides with the Brauer category $\mathcal{B}=\left(\mathbb{N}_{0}, k J(n, m), *\right)$ from $(1.7)$. 
(2.19) A pair partition is plane if it has a frame drawn picture [as in (2.8)] without crossings of lines. We write $T(n, m)$ for the subset of plane pair partitions (TL partitions) and $\mathcal{T}=$ $\left(\mathbb{N}_{0}, k T(n, m), *\right)$ for the corresponding Temperley-Lieb subcategory of $\mathcal{B}$.

\subsection{Paths and the height of a picture/a partition}

Remark 2.20 Fix a rectangle $R$. Each non-self-crossing line $l$ with exterior endpoints in $R$ can be considered to define a separation of $R$ into three parts - the component of $R \backslash l$ containing the left edge of $R$; the other component of $R \backslash l$; and $l$.

(2.21) An alcove of picture $(V, \lambda, L)$ is a connected component of $R \backslash L$.

(2.22) A simple point of a subset $U$ of $\mathbb{R}^{2}$ is a point having a neighbourhood in $U$ that is an open straight segment. (For example in some picture $d=(V, \lambda, L)$, with $L=p(\epsilon(g))$, the non-simple points of $L \backslash \lambda(V)$ are the arc-vertices and crossing points.)

Given a picture $(V, \lambda, L)$, a path in $(V, \lambda, L)$ is a line $l$ in $R$ such that every $k \in(l) \cap L$ is a simple point of $(l)$ and also a simple point of $L \backslash \lambda(V)$.

Thus a path $l$ in $(V, \lambda, L)$ has a well-defined number of line crossings, $\mathrm{d}_{L}(l)$.

Lemma 2.23 Given a path $l$ in picture $d$ connecting points $x, y \in R$ and a distinct point $z \in(l)$, there is a path $l^{\prime}$ connecting $x, y$ that does not contain $z$.

Proof Since $z \in(l)$ it has a neighbourhood either containing only a segment of $l$; or only a crossing of $l$ with a straight segment of $L$ (by the simple point condition on path $l$ ). If we modify the path inside the neighbourhood by a small polygonal detour around $z$ then the modification is a path in $d$ and does not contain $z$.

Lemma 2.24 For each picture $d$ and $x, y \in R$ there is a path in $d$ from $x$ to $y$.

Proof Draw a small straight line $l_{1}$ from $x$ to a point $x^{\prime}$ in an adjacent alcove, choosing $x^{\prime}$ so that the tangent of the straight line $x^{\prime}-y$ is not in the finite set of tangents of segments of lines of $L$; and the line does not contain any element of the finite set of crossing points of $d$. Then $x-x^{\prime}-y$ is a path.

(2.25) By (2.22) and Lemma 2.24 we have established that the notion of distance $\mathrm{d}_{d}(x, y)$ in (1.8) is well-defined. For our notion of height we will need only distances from a certain reference point. Given a picture $d=(V, \lambda, L)$, and points $r, x$ in $R$, the $x$-height $\mathrm{d}_{d}(r, x)$ (or $\mathrm{d}_{L}(r, x)$ ) of $r$ is the minimum value of $\mathrm{d}_{L}(l)$, as defined in (2.22), over paths $l$ in $d$ from $r$ to $x$.

We suppose that $L$ does not intersect the left edge $R_{L}$ of $R$. The (left)-height ht ${ }_{L}(r)=$ $\mathrm{d}_{L}(r, x)$ in case $x$ is any point on $R_{L}$. (Note that this is well-defined.)

The (left)-height of an alcove $A$ is the left-height of a point in $A$. See Fig. 4 for examples.

(2.26) Given a picture $d=(V, \lambda, L)$, recall $\chi(d)$ is the set of crossing points of lines in $d$ [recall from (1.3) that, vertex points aside, lines in $d$ only meet at crossing points]. The left-height $\mathrm{ht}(d)$ is the greatest of the left-heights of the points $x \in \chi(d)$; or is defined to be -1 if there are no crossings.

For example, $d_{1}$ in Fig. 4 has left-height 2 ; and $d_{2}$ has left-height -1 .

(2.27) Finally we say that a partition $p \in P(n, m)$ has left-height ht $(p)=l$ if it has a frame drawn picture of left-height $l$, and no such picture of lower left-height. For example, both pictures in Fig. 4 give the same partition $p$, so ht $(p)=-1$. 

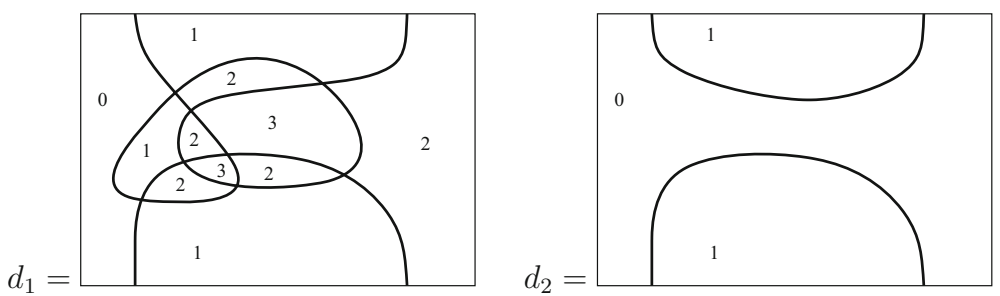

Fig. 4 Example pictures with left-heights of alcoves. (Remark: By $[60, \S 6]$ piecewise linear and piecewise smooth lines are effectively indistinguishable as far as physically drawn figures are concerned)

Since every $p$ has a picture, it will now be clear that $p \rightarrow \operatorname{ht}(p)$ defines a function

$$
\text { ht: } P(n, m) \rightarrow\{-1,0,1,2, \ldots\} .
$$

A path realising the left-height of a point in a picture is called a low-height path. A picture realising the left-height of a partition is called a low-height picture.

(2.28) Define $P_{l}(n, m)$ as the subset of partitions in $P(n, m)$ of left-height $l$, and

$$
P_{\leq l}(n, m)=\bigcup_{j \leq l} P_{j}(n, m) .
$$

Define $J_{l}(n, m)$ as the corresponding subset of $J(n, m)$, and $J_{\leq l}(n, m)$ analogously.

Example 2.29 Here we give the $J_{l}(3,3)$ subsets of $J(3,3)$. Each element is represented by a low-height picture (of course, other pictures could have been chosen instead). Note that it is a Proposition that a given picture is low-height. (In our examples this can be proved using the Jordan curve Theorem. In general it is not so easy, but we will only need existence of low-height pictures, which is clear.) One should keep in mind that the elements of $J_{l}(3,3)$ are pair partitions, not pictures!

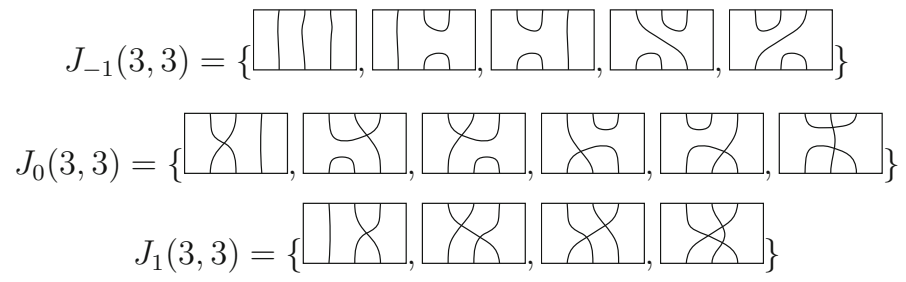

More generally the Brauer algebra identity element $1_{n} \in J_{-1}(n, n)$; the symmetric group Coxeter generator $\sigma_{1} \in J_{0}(n, n) ; \sigma_{i} \in J_{i-1}(n, n)$;

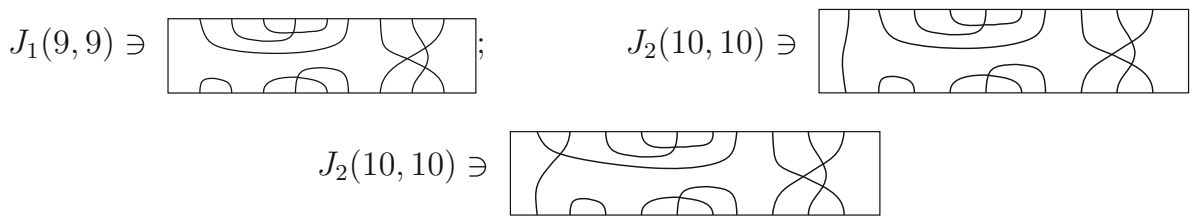

Remark 2.30 Observe that for $p \in J(n, n), \operatorname{ht}(p) \leq n-2$. Hence, in particular, $J_{\leq r}(n, n)=$ $J_{\leq n-2}(n, n)$ for any $n-2<r<\infty$. 
Lemma 2.31 Removing part or all of a line from a picture cannot produce a picture with higher height.

Proof The number of crossings of a path cannot be increased by removing a line. Let $d^{\prime}$ come from $d$ by removing a line. Each crossing point that occurs in $d^{\prime}$ was in $d$, and so its height cannot increase.

(2.32) In particular, if a line has a self-crossing then we can 'short-circuit' the path without increasing the height, or changing the partition. The self-crossing point becomes an arc-vertex with a regular neighbourhood, so the regularity of the picture remains to hold. Thus for each low-height picture there is a low-height picture without line self-crossings.

\section{Algebraic structures over $J_{\leq l}(n, n)$}

We denote by $\mathrm{k}$ the commutative ring $\mathrm{k}=\mathbb{Z}[\delta]$, where $\delta$ is an indeterminate; and $\mathbb{k}=$ $\mathbb{Z}\left[\delta, \delta^{-1}\right]$.

(3.1) Recall the Brauer category $\mathcal{B}$ from (1.7) and consider the case of the ground ring k. Thus for each $n$ we have the Brauer k-algebra $B_{n}^{\mathrm{k}}=\mathrm{kJ}(n, n)$. Recall (e.g. from [10] or Lemma 2.18) that the multiplication in $B_{n}^{\mathrm{k}}$ may be defined via vertical juxtaposition of representative diagrams.

(3.2) Define $J_{l, n}^{\mathrm{k}}$ as the k-subalgebra of $B_{n}^{\mathrm{k}}$ generated by $J_{\leq l}(n, n)$. For $k$ a fixed commutative ring and $\delta_{c} \in k$ we write $J_{l, n}=J_{l, n}\left(\delta_{c}\right)$ for the base-change:

$$
J_{l, n}\left(\delta_{c}\right):=k \otimes_{\mathrm{k}} J_{l, n}^{\mathrm{k}}
$$

(i.e., regarding $k$ as a k-algebra in which $\delta$ acts as $\delta_{c}$ ). For example $J_{l, n}(1)$ is the monoid $k$-algebra for the submonoid of the Brauer monoid associated to $B_{n}(1)$ [59].

(3.3) Define $\mathcal{J}_{l}$ as the subcategory of $\mathcal{B}$ generated by the sets $J_{\leq l}(n, m)$, over all $n, m \in \mathbb{N}_{0}$ (that is, the smallest k-linear subcategory such that the collection of arrows $\mathcal{J}_{l}(n, m)$ contains $J_{\leq l}(n, m)$ for each $\left.n, m\right)$.

Note that the smallest possible height for a crossing is 0 . Thus, noting (2.26), $J_{-1}(n, m)$ denotes the subset with no crossings. That is $J_{-1}(n, m)=T(n, m)$ from (2.19). It will be clear then that $J_{-1, n}^{\mathrm{k}}$ is the Temperley-Lieb algebra. Complementarily, $J_{\leq \infty}(n, m)=J(n, m)$, so $J_{n-2, n}^{\mathrm{k}}\left(=J_{\infty, n}^{\mathrm{k}}\right.$, by Remark 2.30) is the Brauer algebra. Next we will show (our first main theorem) that the 'interpolation' by varying $l$ between these is proper in the following sense:

Theorem 3.4 (I) The sets $J_{\leq l}(n, m)$ form a basis for the k-linear category $\mathcal{J}_{l}$. That is, $\mathcal{J}_{l}=$ $\left(\mathbb{N}_{0}, k J_{\leq l}(n, m), *\right)$. (II) The set $J_{\leq l}(n, n)$ is a $k$-basis for $J_{l, n}^{k}$.

Proof (I) Recall from definition (3.3) that the $n, m$-arrow set in $\mathcal{J}_{l}$ is generated by $J_{\leq l}(n, m)$. Note that $J_{\leq l}(n, m)$ is linearly independent over $\mathrm{k}$ in $\mathcal{J}_{l}$, as it is linearly independent in the Brauer category $\mathcal{B}$. It is thus enough to show that $J_{\leq l}(n, m) \times J_{\leq l}(m, j)$ maps to $\mathrm{kJ}_{\leq l}(n, j)$ under the Brauer category product. For this, we need to show that for every pair of pairpartitions $\left(p_{1}, p_{2}\right) \in J_{\leq l}(n, m) \times J_{\leq l}(m, j)$, determining a partition $p_{3} \in J(n, j)$, we have $p_{3} \in J_{\leq l}(n, j)$. By (2.27) the pair-partitions $p_{1}, p_{2}$ have composable minimum-height pictures, denoted by $d_{1}, d_{2}$, respectively. By Proposition 2.14 their vertical juxtaposition $d_{1} \mid d_{2}$ gives $p_{3}$, so it is sufficient to show that ht $\left(d_{1} \mid d_{2}\right) \leq l$. 

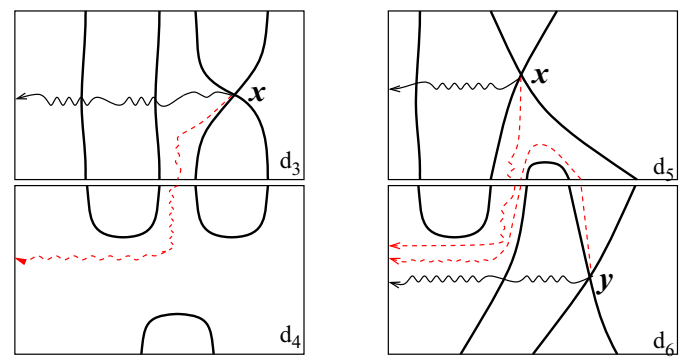

$$
\begin{aligned}
& \operatorname{ht}\left(\mathrm{d}_{3}\right)=2 \\
& \operatorname{ht}\left(\mathrm{d}_{4}\right)=-1 \\
& \operatorname{ht}\left(\mathrm{d}_{3} \mid \mathrm{d}_{4}\right)=0 \\
& \operatorname{ht}\left(\mathrm{d}_{5}\right)=1 \\
& \operatorname{ht}\left(\mathrm{d}_{6}\right)=1 \\
& \operatorname{ht}\left(\mathrm{d}_{5} \mid \mathrm{d}_{6}\right)=0
\end{aligned}
$$

Fig. 5 Examples with heights shown

Observe that by construction the set of crossing points of $d_{1} \mid d_{2}$ is precisely the disjoint union of those of $d_{1}$ and $d_{2}$. Now, also by construction a low-height path from any point $x$ in $d_{1}$ remains a (not necessarily of low height) path in $d_{1} \mid d_{2}$. See the path from $x$ in the figure below for example.
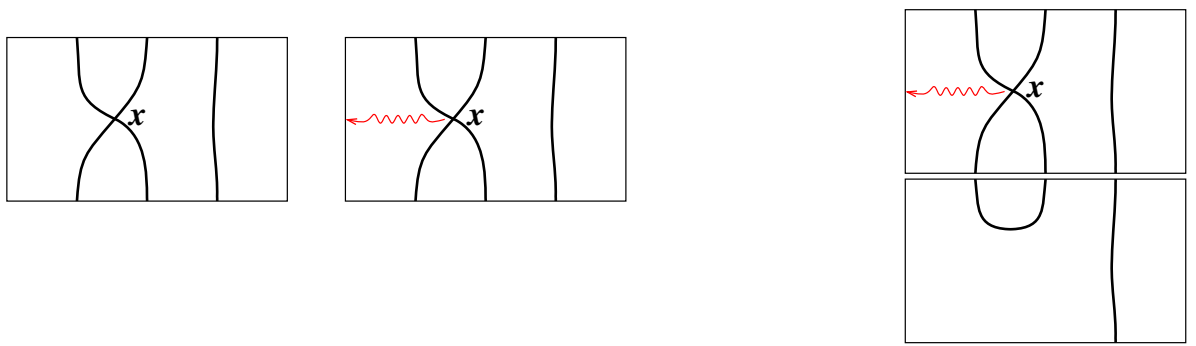

Thus, the left-height of a point (in $d_{1}$ or $d_{2}$ ) cannot increase after concatenation. Hence ht $\left(d_{1} \mid d_{2}\right) \leq l$. (II) follows immediately.

Remark 3.5 As illustrated in Fig. 5, the left-height of $d \mid d^{\prime}$ may be smaller than that of $d$ and $d^{\prime}$, due to paths in $d \mid d^{\prime}$, which are not paths in either $d$ or $d^{\prime}$.

Theorem 3.6 The triple $\mathcal{P}_{l}=\left(\mathbb{N}_{0}, k P_{\leq l}(n, m), *\right)$ is a subcategory of $\mathcal{P}$.

Proof The proof of Theorem 3.4 works mutatis mutandis.

We will discuss connections with known constructions in Sect. 6.2.

\section{Representation theory of $J_{I, n}$}

We now begin to examine the representation theory of $J_{l, n}$. We broadly follow a tower of recollement (ToR) approach [18], extending this as in [32, §6] and [55].

A part in $p \in P(n, m)$ is propagating if it contains both primed and unprimed elements of $\underline{n} \cup \underline{m}^{\prime}$ (see e.g. [48,50]). Write $P(n, l, m)$ for the subset of $P(n, m)$ of partitions with $l$ propagating parts; and similarly $J(n, l, m)$. Define

$$
J_{l}(n, r, m)=J_{l}(n, m) \cap J(n, r, m) .
$$

We write $\#^{\mathrm{P}}(p)$ for the propagating number - the number of propagating parts. 
Define $u$ as the unique element in $J(2,0)$. Note that $\mathcal{P}$ and $\mathcal{B}$ are isomorphic to their respective opposite categories via the opposite mapping $c \mapsto c^{*}$. Thus $u^{*}$ is the unique element in $J(0,2)$. Define $U$ to be the pair partition in $J(2,2)$ determined by the following picture.

$$
U:=u u^{*}=
$$

We use $\otimes$ to denote the monoidal/tensor category composition in $\mathcal{P}$ (that is, the image of the side-by-side concatenation of pictures from (2.16), extended $k$-linearly). For any given $n \geq 2$, set e $=e_{n}$ where

$$
e_{n}=1_{n-2} \otimes U=|||||\ldots| \bigcup_{n} \in J(n, n)
$$

Given a partition $p$ in $P(n, n)$ we write $\left.p\right|_{n-2}$ for the natural restriction to a partition in $P(n-2, n-2)$. (Note that this restriction does not take $J(n, n)$ to $J(n-2, n-2)$.)

\subsection{Index sets for simple $J_{I, n}$-modules}

Here we assume we have base-changed as in (4) to an algebraically closed field $k$. We write $J_{l, n}$ for $J_{l, n}\left(\delta_{c}\right)$ if we do not need to emphasise $\delta_{c}$. Write $k^{\times}$for the group of units.

Proposition 4.1 Suppose $\delta \in k^{\times}$or $n>2$. There is a $k$-module map

$$
\Psi: e J_{l, n} e \cong J_{l, n-2}
$$

given by $\Psi$ : ede $\mapsto$ ede $\left.\right|_{n-2}$. For $\delta \in k^{\times}$this $\Psi$ is an algebra isomorphism.

Let $f=1_{n-3} \otimes U \otimes 1_{1}$ (cf. (5)) and $h=$ ef. Then efe $=e$ and e $J_{l, n}$ ef $=$ ef $J_{l, n}$ ef $=h J_{l, n} h$. For $n>2$, and any $\delta$, there is an algebra isomorphism $h J_{l, n} h \cong J_{l, n-2}$.

Proof Note ede $=d^{\prime} \otimes U$ for some $d^{\prime} \in J_{l, n-2}$, so $\Psi(\mathrm{e} d \mathrm{e})=d^{\prime}$ so the map is injective (just as in the ordinary Brauer case). To show surjectivity in case $\delta \in k^{\times}$consider $d^{\prime}$ in $J_{l, n}$ by the natural inclusion of $J_{l, n-2} \hookrightarrow J_{l, n}$ (the key point here is that the natural inclusion $J_{n-2} \hookrightarrow J_{n}$ takes $J_{l, n-2} \hookrightarrow J_{l, n}$ since the embedding does not change the height of crossings in the $d^{\prime}$ part, as it were, and does not introduce further crossings), so $\Psi\left(\mathrm{e} d^{\prime} \mathrm{e}\right)=\delta d^{\prime}$ for any $d^{\prime} \in J_{l, n-2}$. Other cases are similar.

Corollary 4.2 Suppose that $\Lambda\left(J_{l, n}\right)$ denotes an index set for classes of simple modules of $J_{l, n}$, for any $n$. Then for $n>2$ the set of classes of simple modules $S$ of $J_{l, n}$ such that eS $\neq 0$ may be indexed by $\Lambda\left(J_{l, n-2}\right)$.

Proof Note that $\delta^{-1} \mathrm{e}$ (respectively ef) is idempotent and apply Green's Theorem in [32, $\S 6.2]$.

Corollary 4.3 For $n>2$ the index set $\Lambda\left(J_{l, n}\right)$ may be chosen so that

$$
\Lambda\left(J_{l, n}\right) \backslash \Lambda\left(J_{l, n-2}\right)=\Lambda\left(J_{l, n} /\left(J_{l, n} e J_{l, n}\right)\right)
$$

where $\Lambda\left(J_{l, n} /\left(J_{l, n} e J_{l, n}\right)\right)$ is an index set for simple modules of the quotient algebra by the relation $e=0$ (i.e. the quotient by the ideal $\left.J_{l, n} e J_{l, n}\right)$. In other words $\Lambda\left(J_{l, n}\right) \cong$ $\Lambda\left(J_{l, n-2}\right) \sqcup \Lambda\left(J_{l, n} /\left(J_{l, n} e J_{l, n}\right)\right)$ and the sets $\left\{\Lambda\left(J_{l, n}\right)\right\}_{n}$ are determined iteratively by the sets $\left\{\Lambda\left(J_{l, n} /\left(J_{l, n} e J_{l, n}\right)\right)\right\}_{n}$. 

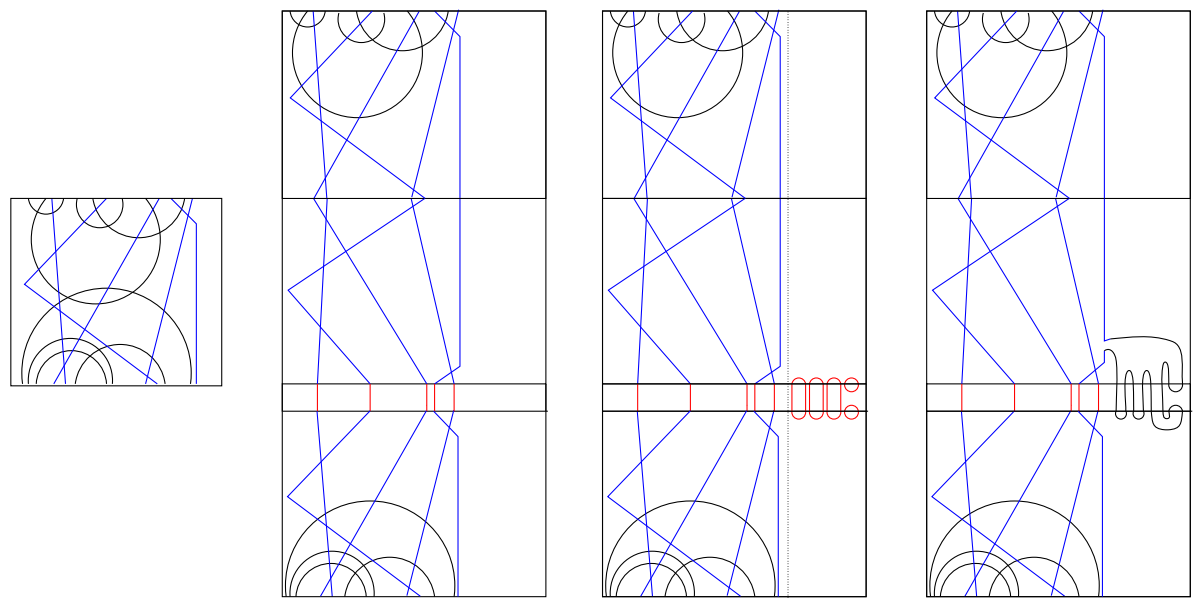

Fig. 6 Writing $p \propto p^{\prime} e p^{\prime \prime}$

Define $J_{l}(n,<m, n)=\cup_{r<m} J_{l}(n, r, n)$ and so on (e.g. $J_{\leq l}(n,<n, n)$ includes every pair partition in $J_{\leq l}(n, n)$ with submaximal number of propagating lines).

Proposition 4.4 The ideal $J_{l, n} e J_{l, n}=k J_{\leq l}(n,<n, n)$ as a $k$-vector space.

Proof Let $p \in J_{\leq l}(n, n)$ have submaximal number of propagating lines, that is $\#^{\mathrm{P}}(p) \leq n-2$ (see page 1258), so that it has at least one northern and one southern pair. Let $d$ be a low-height picture of $p$. We will use $d$ to show that $p \in J_{l, n} \mathrm{e} J_{l, n}$.

For $X$ some subset of $\{N, S\}$, let $d[-X]$ denote the picture obtained from $d$ by deleting the lines from north to north $(N)$, south to south $(S)$, or both. Thus $d[-S]$ is a picture of some $p_{t} \in J(n, j, j)$ where $j=\#^{\mathrm{P}}(p)$. Similarly, abusing notation slightly by writing $u^{*}$ for some low-height picture of $u^{*}$, then $d[-S] \otimes u^{*}$ is a picture of some $p^{\prime} \in J(n, j, j+2)$.

By the Deletion Lemma 2.31 the height of $d[-N]$ does not exceed that of $d$, and similarly for $d[-N S]$ and $d[-S]$. Note that, since $d[-N S]$ is a picture of a permutation (of the propagating lines); and $d[-N S]^{*}$ is a picture of the inverse, we have that in the picture category (Proposition 2.11),

$$
d^{\prime}=d[-S]\left|d[-N S]^{*}\right| d[-N]
$$

is another picture for $p$. (An example is provided by Fig. 6: the original picture of $p$ is on the left, whereas $d[-S]\left|d[-N S]^{*}\right| d[-N]$ is on the center-left.)

Next, observe that one can add some loops on the right of this picture, (red circles in the example Fig. 6) with no change to the height of the picture, or the resulting partition $p$. Thus, up to an overall factor of a power of $\delta, p$ can be expressed in the form $p^{\prime} \mathrm{e} p^{\prime \prime}$ where a picture for $p^{\prime}$ is $\left(d[-S] \mid d[-N S]^{*}\right) \otimes\left(u^{*}\right)^{\otimes(n-j) / 2}$ (a picture of height $\leq l$ by construction), and $p^{\prime \prime}$ is $d[-N] \otimes u^{\otimes(n-j) / 2}$, and hence $p^{\prime}, p^{\prime \prime} \in J_{l, n}$. Finally note that the red loops can be replaced by suitable non-crossing deformations of lines from above and below (cf. the rightmost picture in example Fig. 6).

(4.5) By Proposition 4.4 the quotient algebra $J_{l, n} /\left(J_{l, n} \mathrm{e} J_{l, n}\right)$ has a basis which is the image of $J_{\leq l}(n, n, n)$. Note that these elements of $J_{l, n}$ form a subgroup as well as their image spanning a quotient algebra. 
For $n<l+2$ this group is isomorphic to $S_{n}$; otherwise it is isomorphic to $S_{l+2}$ (since there can be no crossings after the first $l+2$ lines). The quotient itself is then the corresponding group algebra. That is, $\Lambda\left(J_{l, n} /\left(J_{l, n} \mathrm{e} J_{l, n}\right)\right) \cong \Lambda\left(k S_{\min (n, l+2)}\right)$, where $\Lambda\left(k S_{n}\right)$ denotes an index set for simple $k S_{n}$-modules, which we can take to be the set of char. $k$-regular integer partitions [35]. Combining with Proposition 4.3, we thus have the following.

Theorem 4.6 Let $\Lambda_{n}$ denote the set of integer partitions of $n$ and $\Lambda_{n, k}$ the subset of char.kregular partitions as in [35] (so that $\Lambda\left(\mathbb{C} S_{n}\right)=\Lambda_{n}$ for any $n$ and $\Lambda\left(k S_{n}\right)=\Lambda_{n, k}$ for any $k)$. Let $\Lambda_{n}^{(p)}:=\{p\} \times \Lambda_{n}$ (so that $\Lambda_{n}^{(1)}$ and $\Lambda_{n}^{(2)}$ are disjoint copies of $\left.\Lambda_{n}\right)$, and similarly for $\Lambda_{n, k}$. Define $\Gamma_{n}=\{n, n-2, \ldots, 1 / 0\}$. Then, with $\delta \neq 0$,

$$
\Lambda\left(J_{l, n}\right)=\bigcup_{p \in \Gamma_{n}} \Lambda_{\min (p, l+2), k}^{(p)}
$$

and specifically over $k=\mathbb{C}$, with $\delta \neq 0$,

$$
\Lambda\left(J_{l, n}\right) \stackrel{k=\mathbb{C}}{=} \bigcup_{p \in \Gamma_{n}} \Lambda_{\min (p, l+2)}^{(p)} \cong\left(\bigcup_{p=0}^{l+1} \Lambda_{p}\right) \cup\left(\bigsqcup_{p=l+2}^{n} \Lambda_{l+2}\right)
$$

where $\bigcup_{p}^{\prime}$ denotes a range including only $p$ congruent to $n$ mod. 2 ; and $\bigsqcup_{p}{ }^{\prime} \Lambda_{l+2}$ denotes disjoint copies of $\Lambda_{l+2}$ with the same constraint on the range. For $\delta=0$ the result is the same except to omit the integer partition of 0 when $n$ is even.

Proof The cases not covered by Proposition 4.3 are for $n \leq 2$ (in particular when $\delta=0$ ). These are simple direct calculations (cf. [55]).

(4.7) Let $J^{\|}(n, l, m)$ denote the subset of $J(n, l, m)$ of elements $p$ having a picture $d$ for which $d[-N S]$ has no crossings. Recall (e.g. from [55]) the polar decomposition of an element of $J(n, m, n)$ : the inverse of the map $v: J^{\|}(n, m, m) \times J(m, m, m) \times J^{\|}(m, m, n) \stackrel{\sim}{\rightarrow}$ $J(n, m, n)$, given by the category composition. Note that if $p \in J_{\leq l}(n, m, n)$ then $l$ bounds the height of all three factors in the polar decomposition. (The argument is analogous to the argument at (6). Firstly note that if $d$ is a low-height picture of $p$ then $d[-S] \mid d[-N S]^{*}$ is a picture of the northern polar factor of no higher height. The other factors are similar.) That is, the restriction $v^{-1}: J_{\leq l}(n, m, n) \hookrightarrow J_{\leq l}^{\|}(n, m, m) \times J_{\leq l}(m, m, m) \times J_{\leq l}^{\|}(m, m, n)$. On the other hand the image of $v$ on this codomain lies in $J_{\leq l}(n, m, n)$ by Theorem 3.4, so the restriction as given is a bijection.

\subsection{On quasihereditary cases of $J_{l, n}$}

The proof of the main result of this section (Theorem 4.12) follows closely the Brauer algebra case, as for example in [19]. We focus mainly on the new features required for the present case.

(4.8) For $n \geq 2 t$ define $e_{n, t}=1_{n-2 t} \otimes U^{\otimes t}$ and (when $\delta \in k^{\times}$) $e_{n, t}^{\prime}=\delta^{-t} e_{n, t}$. Note that $e_{n, t} \in J_{-1}(n, n-2 t, n)$. For example $\mathrm{e}=e_{n, 1}$ and

$$
e_{n, 2}=||+\mid \cap \cap
$$

We have the following useful corollary to the proof of Proposition 4.4. 
Corollary 4.9 The ideal $J_{l, n} e_{n, t} J_{l, n}=k J_{\leq l}(n, \leq n-2 t, n)$ as a $k$-vector space.

Proof The proof is the same as for Proposition 4.4 except that we use a different 'meandering' deformation of the path in the final stage of the construction of $d \in J_{l}(n, \leq n-2 t, n)$, as in Fig. 6. This is needed to avoid closed loops in the construction using $e_{n, t}$. Specifically we change, for example, from the first to the second here:
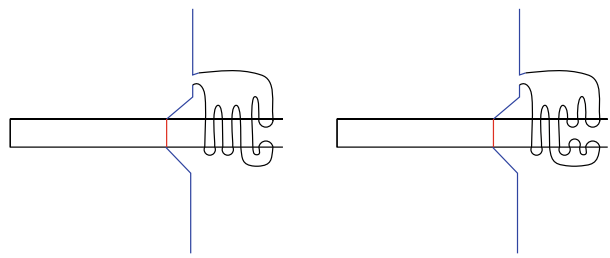

Note that there is room for enough meanders because of the bound on the number of propagating lines in $d$.

(4.10) Define the quotient algebra

$$
J_{l, n, t}=J_{l, n} / J_{l, n} e_{n, t+1} J_{l, n}
$$

By Corollary 4.9 this algebra has basis $J_{\leq l}(n, \geq n-2 t, n)$.

Proposition 4.11 For each triple $n, l, t$ the following hold when $\delta \in k^{\times}$.

(i) The algebra $A=e_{n, t} J_{l, n, t} e_{n, t}$ is isomorphic to $k S_{\min (n-2 t, l+2)}$.

(ii) The multiplication map $J_{l, n, t} e_{n, t} \otimes_{A} e_{n, t} J_{l, n, t} \stackrel{\mu}{\rightarrow} J_{l, n, t} e_{n, t} J_{l, n, t}$ is a bijection of $\left(J_{l, n, t}, J_{l, n, t}\right)$-bimodules.

Proof (i) The number of propagating lines must be at least $n-2 t$, but with the $e_{n, t}$ 's present this is also the most it can be, so every propagating line in $e_{n, t}$ is propagating in $A$, and indeed $A$ is isomorphic to the group algebra of a symmetric group. The group is $S_{\min (n-2 t, l+2)}$ by the height condition.

(ii) The map is clearly surjective. We construct an inverse using the polar decomposition (4.7). Note that $J_{l, n, t} e_{n, t}$ has a basis in bijection with $J_{\leq l}(n, n-2 t, n-2 t) \cong J_{\leq l}^{\|}(n, n-$ $2 t, n-2 t) \times J_{\leq l}(n-2 t, n-2 t, n-2 t)$. Subset $e_{n, t} J_{l, n, t}$ can be treated similarly. It then follows from the definition of the tensor product that the left-hand side has a spanning set whose image is independent on the right.

Theorem 4.12 If $\delta$ invertible in $k=\mathbb{C}$ then $J_{l, n}$ is quasihereditary, with heredity chain given by $\left(1, e_{n, 1}^{\prime}, e_{n, 2}^{\prime}, \ldots\right)$ as defined in (4.8).

Proof Noting that $\mathbb{C} S_{\min (n-2 t, l+2)}$ is semisimple, this follows immediately from Proposition 4.11. Specifically Proposition 4.11 shows, via e.g. [22, Statement 7], that $\left(1, e_{n, 1}^{\prime}, e_{n, 2}^{\prime}, \ldots\right)$ gives a heredity chain in the sense of $[16,21]$ (to strictly match their conventions, one reverses the order of elements). Cf. e.g. [19,24].

\subsection{Aside: slick proof of quasiheredity in the monoid case}

Note that for $a \in J(n, n)$ we have $a a^{*} a=a$ in $J_{l, n}$. Since the height of $a^{*}$ is the same as $a$ we have the following. 
Proposition 4.13 The algebra $J_{l, n}(1)$ is a regular-monoid $k$-algebra (i.e. $a \in$ a $J_{l, n}$ a for all $\left.a \in J_{l}(n, n)\right)$.

Corollary 4.14 The algebra $J_{l, n}(1)$ is quasihereditary when $k=\mathbb{C}$.

Proof Use Putcha's Theorem [64], that a regular-monoid $\mathbb{C}$-algebra is quasihereditary.

\subsection{Standard modules of $J_{l, n}$}

We may construct a complete set of 'standard modules' for each $J_{l, n}$ as follows. The modules we construct are 'standard' with respect to a number of different compatible axiomatisations (the general idea of standard modules, when such exist, is that they interpolate between simple and indecomposable projective modules). For example (I) we can construct quasihereditary standard modules by enhancing the heredity chain in Theorem 4.12 to a maximal chain cf. [24]; (II) we can construct the modular reductions of lifts of 'generic' irreducible modules in a modular system cf. [6]; (III) we can construct globalisations of suitable modules from lower ranks cf. [42,51,55]. We are mainly interested in a useful upper-unitriangular property of decomposition matrices that we establish in Proposition 4.27.

(4.15) Note that $k J_{\leq l}(n, m)$, with $m \leq n$ say, is a left $J_{l, n}$ right $J_{l, m}$-bimodule, by the category composition. By the bottleneck principle it has a sequence of submodules:

$$
k J_{\leq l}(n, m)=k J_{\leq l}(n, \leq m, m) \supset k J_{\leq l}(n, \leq m-2, m) \supset \cdots
$$

For given $l$, each section

$$
\mathfrak{J}_{n, m}^{p}:=k J_{\leq l}(n, \leq p, m) / k J_{\leq l}(n, \leq p-2, m)
$$

thus has basis $J_{\leq l}(n, p, m)$. In particular the top section has basis $J_{\leq l}(n, m, m)$.

(4.16) The above holds in particular for the case $m=n$, where our sequence is an ideal filtration of the algebra, cf. Corollary 4.9. Define the quotient algebra

$$
J_{l, n}^{/ p}=J_{l, n} / k J_{l}(n, \leq p, n) .
$$

Note that this is the same as $J_{l, n, t}$ with $p+2=n-2 t$ (but now without restriction on $\delta$ ). The index $p$ tells us that partitions with $p$ or fewer propagating lines are congruent to zero in the quotient.

(4.17) In particular, as noted in (4.5), $J_{l, n}^{/ n-2} \cong k S_{\min (n, l+2)}$. Specifically $J_{l, n}^{/ n-2}$ has basis $J_{\leq l}(n, n, n)$, which is of the form $J(l+2, l+2, l+2) \otimes 1_{n-(l+2)}$, i.e. $S_{l+2} \otimes 1_{n-(l+2)}$, when $n>l+2$ (since there can be no crossing lines after the first $l+2$ in this case).

(4.18) Note that $\mathfrak{J}_{n, m}^{p}$ is also a left $J_{l, n}^{/ p-2}$ right $J_{l, m}^{/ p-2}$ bimodule. Thus we have a functor

$$
\mathfrak{J}_{n, m}^{p} \otimes_{J_{l, m}^{\prime p-2}}-: J_{l, m}^{/ p-2}-\bmod \rightarrow J_{l, n}^{/ p-2}-\bmod
$$

and in particular a functor

$$
\begin{aligned}
& \mathfrak{J}_{n, p}^{p} \otimes_{k S_{p}}-: k S_{p}-\bmod \rightarrow J_{l, n}^{/ p-2}-\bmod \quad(p \leq l+2) \\
& \mathfrak{J}_{n, p}^{p} \otimes_{k S_{l+2}}-: k S_{l+2}-\bmod \rightarrow J_{l, n}^{/ p-2}-\bmod \quad(p>l+2)
\end{aligned}
$$

(in case $p>l+2$, the right action of $k S_{p_{l}}=k S_{l+2}$ in the form $k S_{l+2} \otimes 1_{p-l-2}$ is understood). 
(4.19) With this functor in mind we recall some facts about the symmetric groups. For any symmetric group $S_{m}$ and a partition $\lambda \vdash m$, let $\mathcal{S}_{\lambda}$ denote the corresponding Specht module of $S_{m}$-see e.g. [35,36]. Recall that there is an element $\epsilon_{\lambda}$ in $k S_{m}$ such that $\mathcal{S}_{\lambda}=k S_{m} \epsilon_{\lambda}$. If $k \supset \mathbb{Q}$ then $\epsilon_{\lambda}$ may be chosen idempotent. Example: The element $\epsilon_{(2)} \in k S_{2}$ is unique up to scalars: $\epsilon_{(2)}=1_{2}+\sigma_{1}$ (in the obvious notation). Thus a basis for $\mathcal{S}_{(2)}$ is $b_{(2)}=\left\{\epsilon_{(2)}\right\}$.

(4.20) Recall that, for given $k, \Lambda\left(J_{l, n}\right)$ denotes the index set for simple modules as in Theorem 4.6. Let us also define a set

$$
\Lambda^{-}\left(J_{l, n}\right):=\bigcup_{p \in \Gamma_{n}} \Lambda_{\min (p, l+2), k}^{(p)}
$$

as in Theorem 4.6, independently of $k$. (Note that for $k=\mathbb{C}$ and $\delta \neq 0$ we have $\Lambda\left(J_{l, n}\right)=$ $\Lambda^{-}\left(J_{l, n}\right)$.)

For given $l$ define $p_{l}:=\min (p, l+2)$. For any $p$ and $\lambda \vdash p_{l}$, that is for $(p, \lambda) \in \Lambda^{-}\left(J_{l, n}\right)$, we define a global-standard $J_{l, n}$-module using (4.18)

$$
\Delta_{p, \lambda}^{n}=\Delta_{p, \lambda}=\mathfrak{J}_{n, p}^{p} \otimes_{k S_{p_{l}}} \mathcal{S}_{\lambda} .
$$

(4.21) For given $l, p$ let $\lambda \vdash p_{l}$. We can consider $k S_{p_{l}} \hookrightarrow k J\left(p_{l}, p_{l}\right)$, indeed $k S_{p_{l}} \stackrel{\sim}{\rightarrow}$ $k J_{\leq l}\left(p_{l}, p_{l}, p_{l}\right)$ by the inclusion in (4.5), and hence $\epsilon_{\lambda} \in k J_{\leq l}\left(p_{l}, p_{l}, p_{l}\right)$. Thus

$$
E_{n}(p, \lambda):=\epsilon_{\lambda} \otimes 1_{p-p_{l}} \otimes U^{\otimes(n-p) / 2}
$$

lies in $J_{l, n}$. For any $\epsilon_{\lambda}$ we may write the image in $\mathcal{P}$ schematically as $\lambda$ with $2 \times|\lambda|$ legs; e.g. (2) with two pairs of legs. Thus for example with $l=1$ we have:

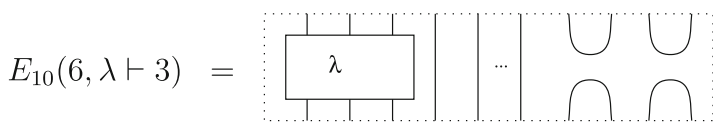

Define another $J_{l, n}$-module by

$$
D_{p, \lambda}=J_{l, n}^{/ p-2} E_{n}(p, \lambda)
$$

Proposition If $k \supset \mathbb{Q}$ and $\delta \in k^{\times}$then the elements $E_{n}(p, \lambda)$ are (unnormalised) idempotents serving to refine the chain $\left(1, e_{n, 1}^{\prime}, e_{n, 2}^{\prime}, \ldots\right)$ in Theorem 4.12 and $\left\{D_{p, \lambda} \mid(p, \lambda) \in\right.$ $\left.\Lambda\left(J_{l, n}\right)\right\}$ are the quasihereditary-standard modules $[24, \S \mathrm{A} 1]$ of the quasihereditary structure in Theorem 4.12.

Proof Let $A$ be a quasihereditary algebra with heredity chain $e^{-}=\left(e^{1}, e^{2}, \ldots, e^{i}, \ldots, e^{l}\right)$; and define $A^{i}=A / A e^{i+1} A$. To refine towards a maximal heredity chain from heredity chain $e^{-}$we first decompose each $e^{i}$ into a primitive idempotent decomposition of it regarded as the identity in $e^{i} A^{i} e^{i}$ (cf. e.g. [24, §A3]). Let $e_{\lambda}$ be any idempotent in this decomposition ( $\lambda$ labeling a simple module of $e^{i} A^{i} e^{i}$ ) that lifts from $A^{i}$ to $A$. Then the lift $e^{\lambda}$ can be the first idempotent in the refinement at 'level' $i$ (again cf. [24]). In our case, comparing the construction with Proposition 4.11 we see that each of the $E_{n}(p, \lambda)$ 's provides such an idempotent.

Subsequent steps in a full chain refinement involve partial sums of such idempotents. But since any order may be used to introducing these into the chain (within level $i$ ), and $\Delta(\lambda)$ is independent of this choice [24], then $\Delta(\lambda)=A^{i} e_{\lambda}$ may be taken to be the corresponding standard module. 
Example For any $l$, a basis of $D_{2,(2)}$ for $n=4$ is in bijection with $J_{\leq l}^{\|}(4,2,2)$ (as in 4.7). Indeed, omitting an arc in the bottom-right that is irrelevant to the algebra action:

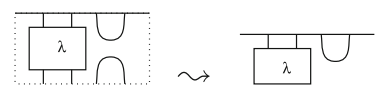

the full basis for $l>0$ may be depicted:
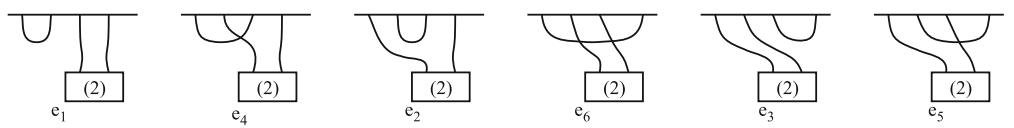

Note that the action of the algebra in this depiction is from above. Note that, if $l=0$, then $J_{\leq 0}^{\|}(4,2,2)$ is smaller.

Proposition 4.22 Let $(p, \lambda) \in \Lambda^{-}\left(J_{l, n}\right)$, so $\lambda \vdash p_{l}$, and fix a basis $b_{\lambda}$ for $\mathcal{S}_{\lambda}=k S_{p_{l}} \epsilon_{\lambda}$, as in $[35, \S 4]$. Let

$$
B_{p, \lambda}=\left\{x\left(y \otimes 1_{p-p_{l}}\right) \mid x \in J_{\leq l}^{\|}(n, p, p) ; y \in b_{\lambda}\right\}
$$

regarded as a subset of $\mathfrak{J}_{n, p}^{p}$. Then $B_{p, \lambda}$ gives a basis for the $J_{l, n}$-module $D_{p, \lambda}$ up to isomorphism (in which $(n-p) / 2$ irrelevant arcs are omitted as in (7)).

Proof By definition $D_{p, \lambda}=J_{l, n}^{/ p-2} E_{n}(p, \lambda)$ is spanned by elements $x\left(y \otimes 1_{p-p_{l}}\right)$ as in $B_{p, \lambda}$ except with $x \in J_{\leq l}(n, p, p)$ (again ignoring irrelevant arcs in the bottom-right). Thus we need to show that we can omit $x$ 's with crossing propagating lines without breaking the spanning property. Some example elements of $D_{5, \lambda}$ with $p>l+2$ (in case $p=5, l=1$ ) provide a useful visualisation here:
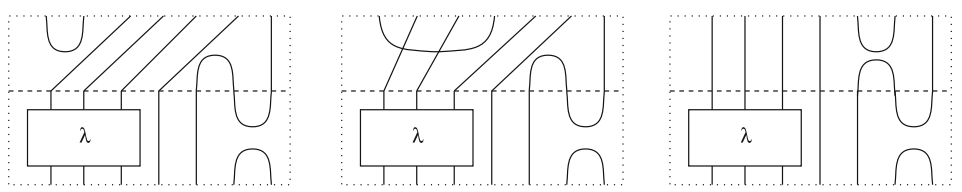

First note that a basis element must have $p$ propagating lines by the quotient. Any crossing in the first $\min (p, l+2)$ of these can be 'absorbed' by the $b_{\lambda}$ part of the basis. There cannot be a crossing in any remaining propagating lines by the height restriction, cf. (4.7).

Corollary $4.23 D_{p, \lambda} \cong \Delta_{p, \lambda}$.

Proof Compare our basis $B_{p, \lambda}$ in (4.22) above with the construction for $\Delta_{p, \lambda}$ in (4.20). The main difference is combination via $\otimes_{k} S_{p_{l}}$ rather than multiplication. This gives us a surjective map right to left. One then compares dimensions.

Proposition 4.24 Suppose $k \supseteq \mathbb{Q}$ and either $\delta \neq 0$ or $p \neq 0$. Then for given $l$, $E_{n}(p, \lambda) J_{l, n}^{/ p-2} E_{n}(p, \lambda)=k E_{n}(p, \lambda)$.

Proof Pictorially/schematically we have:

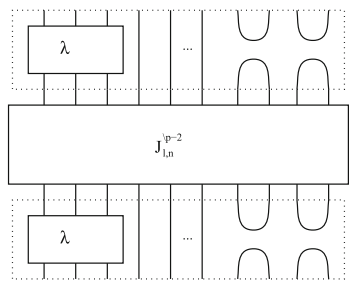

$(4.1),(4.16)$

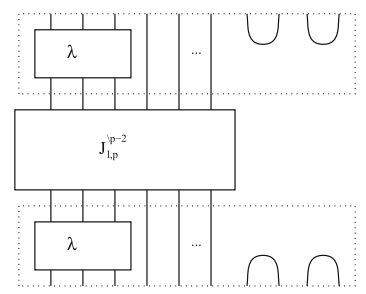

(4.17)

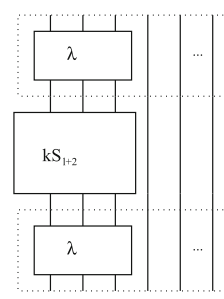


That is, firstly

$$
\begin{aligned}
& \left(\epsilon_{\lambda} \otimes 1_{p-p_{l}} \otimes U^{\otimes(n-p) / 2}\right) J_{l, n}^{/ p-2}\left(\epsilon_{\lambda} \otimes 1_{p-p_{l}} \otimes U^{\otimes(n-p) / 2}\right) \\
& =\left(\left(\epsilon_{\lambda} \otimes 1_{p-p_{l}}\right) J_{l, p}^{/ p-2}\left(\epsilon_{\lambda} \otimes 1_{p-p_{l}}\right)\right) \otimes U^{\otimes(n-p) / 2}
\end{aligned}
$$

by Proposition 4.1. But then $J_{l, p}^{/ p-2} \cong k S_{\min (p, l+2)}$ by (4.17). Finally $\epsilon_{\lambda} k S_{\min (p, l+2)} \epsilon_{\lambda}=k \epsilon_{\lambda}$ by the Specht property (4.19) [36], which implies that $\epsilon_{\lambda}$ is primitive idempotent in $k S_{|\lambda|}$ when $k S_{|\lambda|}$ is semisimple (and so for example when $k \supseteq \mathbb{Q}$ ).

Corollary 4.25 Suppose $k \supseteq \mathbb{Q}$ and either $\delta \neq 0$ or $p \neq 0$. Then for $(p, \lambda) \in \Lambda\left(J_{l, n}\right), D_{p, \lambda}$ is indecomposable projective as a $J_{l, n}^{/ p-2}$-module; and hence indecomposable with simple head as a $J_{l, n}$-module.

Note the natural inclusion of $J_{l, n-2}$ in $J_{l, n}$. Note that this $J_{l, n-2}$ commutes with $e_{n}$. Thus given a left $J_{l, n}$-module $M$ then $e_{n} M$ is a left $J_{l, n-2}$-module, and similarly on the right. In particular $J_{l, n} e_{n}$ is a right- $J_{l, n-2}$-module (isomorphic as such to $k J_{\leq l}(n, n-2)$ ). Alternatively, using Proposition 4.1 both $J_{l, n} e_{n}$ (when $\delta \neq 0$ ) and $J_{l, n} \mathrm{~h}$ (when $n>2$ ) are right $J_{l, n-2}$-modules by 'idempotent inclusion'. The two right-module structures on $J_{l, n} e_{n}$ are isomorphic, when the second is defined. First using $J_{l, n} e_{n}$ then, for each $n$ we have also the pair of functors

$$
J_{l, n-2}-\bmod \underset{F_{e}}{\stackrel{G_{e}}{\rightleftarrows}} J_{l, n}-\bmod
$$

given by $G_{e} M=J_{l, n} e_{n} \otimes_{J_{l, n-2}} M$ and $F_{e} N=e_{n} N$. Similarly for $n-2>0$ we have functors $G_{\mathrm{h}}$ and $F_{\mathrm{h}}$. By Proposition 4.1 and $[32, \S 6]$ the pair $\left(G_{\mathrm{h}}, F_{\mathrm{h}}\right)$ is adjoint; and the pair $\left(G_{e}, F_{e}\right)$ is adjoint when $\delta \neq 0$.

Proposition 4.26 For $\delta \in k^{\times}$, and for $\delta=0$ with $(n, p) \neq(2,0)$,

$$
G_{e} \Delta_{p, \lambda}^{n} \cong \Delta_{p, \lambda}^{n+2} \text {. }
$$

For $\delta \in k^{\times}$, and for $\delta=0$ with $(n, p, \lambda) \neq(0,2,(2))$,

$$
F_{e} \Delta_{p, \lambda}^{n+2} \cong \begin{cases}\Delta_{p, \lambda}^{n} & p \leq n \\ 0 & p=n+2\end{cases}
$$

For $n>0$ and any $\delta \in k$ the functors $G_{h}$ and $F_{h}$ act as in (9) and (10) respectively.

Proof Consider the functor $G_{m}^{n}: J_{l, m}-\bmod \rightarrow J_{l, n}-\bmod$ given by $M \mapsto k J_{\leq l}(n, m) \otimes J_{l, m}$ $M$. By Corollary 4.23 we have that $\Delta_{p, \lambda}^{n} \cong G_{p}^{n} \mathcal{S}_{\lambda}$ (with $\mathcal{S}_{\lambda}$ understood as a $\bar{J}_{l, p}$-module in the obvious way). From the definition of the $G_{e}$ functor note then that the first result follows if the multiplication map $k J_{\leq l}(n, m) \otimes_{J_{l, m}} k J_{\leq l}(m, p) \rightarrow k J_{\leq l}(n, p)$ is an isomorphism for $n>m>p$. This holds, by a routine calculation, except in case $m=2$ with $\delta=0$. (These manipulations are essentially the same as in the Brauer case, see e.g. [19,54,55]. In this proposition our new geometrical constraints do not affect the argument.) The $G_{\mathrm{h}}$ and $F$ cases are similar.

Note that $G_{\mathrm{h}}$ is right exact and preserves projectivity and indecomposability (see e.g. [32, $\S 6])$. It follows that it preserves the simple-head property. Iterating, it follows that $\Delta_{p, \lambda}$ is indecomposable when $\mathcal{S}_{\lambda}$ is, i.e. when char. $k \neq 2$ [35, cor.13.18]. Similarly it follows that $\Delta_{p, \lambda}$ has simple head when $\mathcal{S}_{\lambda}$ has, i.e. when $\lambda$ is char.k-regular (in the sense of [35]). The 
case not covered by $G_{\mathrm{h}}$ or $G_{e}$ is $\lambda=\emptyset$ when $\delta=0$ and $n=2$. But here $\Delta_{0, \emptyset}$ is simple and hence simple head by direct calculation (one proceeds by direct analogy with [55]).

For $k$ as in (4), a field that is a k-algebra by giving a value $\delta \in k$, let $\Lambda^{k}\left(J_{l, n}\right)$ denote the subset of $\Lambda^{-}\left(J_{l, n}\right)$ of pairs $(p, \lambda)$ in which $\lambda$ is char. $k$-regular (and if $\delta=0$ then $p \neq 0$ ). Thus $\Lambda\left(J_{l, n}\right)=\Lambda^{k}\left(J_{l, n}\right)$ for any given $k$, by Theorem 4.6 , and in particular $\Lambda \mathbb{C}_{\left(J_{l, n}\right)=\Lambda^{-}\left(J_{l, n}\right)}$ (when $\delta \neq 0$ ). Fixing $k$, for $(p, \lambda) \in \Lambda^{k}\left(J_{l, n}\right)$ we can now write $L_{p, \lambda}=$ head $\left(\Delta_{p, \lambda}\right)$ for the simple head (see e.g. [6, §1.2]). Note from (10) that these are pairwise non-isomorphic. Write $\left[\Delta_{p, \lambda}: L_{p^{\prime}, \lambda^{\prime}}\right]$ for the multiplicity of $L_{p^{\prime}, \lambda^{\prime}}$ as a composition factor in $\Delta_{p, \lambda}$; and $C^{\Delta}=\left(\left[\Delta_{p, \lambda}: L_{p^{\prime}, \lambda^{\prime}}\right]\right)_{(p, \lambda),\left(p^{\prime}, \lambda^{\prime}\right)}$ for the corresponding decomposition matrix.

Proposition 4.27 (I) The modules $\left\{\Delta_{p, \lambda}\right\}$ are a complete set of standard modules of $J_{l, n}$ in the quasihereditary algebra cases ( $\delta$ invertible and char. $>l+2$, for example $k=\mathbb{C}$ ). (II) The simple decomposition matrix $C^{\Delta}$ for this set of modules is upper unitriangular (when written out in any order so that $(p, \lambda)>\left(p^{\prime}, \lambda^{\prime}\right)$ when $\left.p>p^{\prime}\right)$. (III) More generally $C^{\Delta}$ may be written in the form

$$
C^{\Delta} \stackrel{n \text { even }}{=}\left(\begin{array}{ccccc}
D^{0} & \cdot & \cdot & & \cdot \\
& D^{2} & \cdot & & \cdot \\
& & \ddots & & \\
& & & D^{\min (n, l+2)}
\end{array}\right), C^{\Delta} \stackrel{n \text { odd }}{=}\left(\begin{array}{ccccc}
D^{1} & \cdot & \cdot & & \cdot \\
& & D^{3} & \cdot & \\
& & & \ddots & \cdot \\
& & & D^{\min (n, l+2)}
\end{array}\right)
$$

where $D^{n}$ is the decomposition matrix for the Specht modules of $k S_{n}$ (in our case it is natural to order integer partitions in increasing dictionary order, cf. [35]) and $D^{0}=(1)$; blank block-entries denote zero blocks, and dot block-entries are not necessarily zero. If $\delta=0$ then the $D^{0}$ row is omitted.

Proof (I) Follows from Corollary 4.23 and (4.21) Proposition, and the proof of Theorem 4.12. For (II) and (III) note that Proposition 4.26 implies, cf. [32, §6.6], that the composition factors with the same label in $\Delta_{p, \lambda}^{n}$ and $\Delta_{p, \lambda}^{n+2}$ have the same multiplicity. The only possible new factors in $\Delta_{p, \lambda}^{n+2}$ have the property $e_{n+2} S=0$. Applying this iteratively on $n$ gives the claimed result.

\subsection{Towards the Cartan decomposition matrices}

The Cartan decomposition matrix encodes the fundamental invariants of an algebra [6]. Given the difficulty experienced in computing them in case $l=-1$ and particularly $l=\infty$ [55] we can anticipate that they will not be easy to determine in general. However, the main tools used in cases $l=-1$ and $l=\infty$ can be developed in general, as we show next. We start with a corollary to Proposition 4.27(I-II).

For each $\Delta_{p, \lambda}$ we will use a contravariant form as in $[32, \S 2.7]$. The form here is the analogue of the usual form for the Brauer algebra with respect to the involutive antiautomorphism $*[34,55]$. That is, for $e_{i}, e_{j} \in \Delta_{p, \lambda}$, a suitable inner product $\left\langle e_{i}, e_{j}\right\rangle$ is given by $e_{i}^{*} e_{j}=\left\langle e_{i}, e_{j}\right\rangle E_{n}(p, \lambda)$. This is well-defined for $k \supseteq \mathbb{Q}$ by Proposition 4.24. The form rank is given by the matrix rank of the Gram matrix over a basis. 
Example A basis for $\Delta_{2,(2)}^{4}$ is given in (8). In particular then (with $\lambda=(2)$ ):

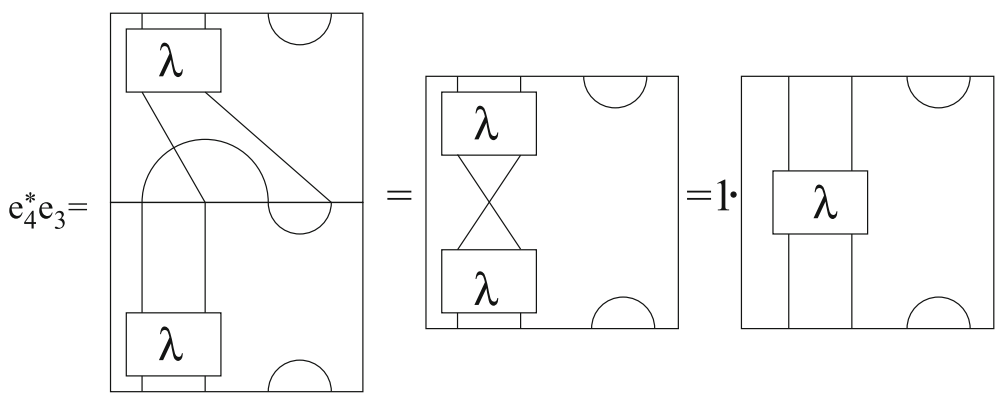

so $\left\langle e_{3}, e_{4}\right\rangle=1$ (noting that $\left.\sigma_{1} \epsilon_{(2)}=\epsilon_{(2)}\right)$.

The form can be generalised to arbitrary $k$, but then the final stage of reduction uses the form on Specht modules from [35], which introduces an integer factor. These factors can be computed, but in our example we focus on characteristic zero.

Corollary 4.28 Fix $k=\mathbb{C}$ and $\delta \in k$. Module $\Delta_{p, \lambda}$ has a contravariant form (with respect to *) that is unique up to scalars. The rank of this form determines the dimension of the simple module $L_{p, \lambda}$.

Proof The space of contravariant forms is in bijection with the space of module maps from $\Delta_{p, \lambda}$ to its contravariant dual (the analogous right-module $E_{n}(p, \lambda) J_{l, n}^{/ p-2}$ treated as a leftmodule via ordinary duality). But by the upper-unitriangular property Proposition 4.27 this space is spanned by any single map from the head to the socle.

In our $\Delta_{2,(2)}^{4}$ example, setting $f_{i, j}:=\left\langle e_{i}, e_{j}\right\rangle$, one computes the Gram matrix

$$
f=\left(\begin{array}{lll|l|ll}
\delta & 1 & 0 & 1 & 1 & 1 \\
1 & \delta & 1 & 1 & 1 & 0 \\
0 & 1 & \delta & 1 & 1 & 1 \\
\hdashline 1 & 1 & 1 & \delta & 0 & 1 \\
\hdashline 1 & 1 & 1 & 0 & \delta & 1 \\
1 & 0 & 1 & 1 & 1 & \delta
\end{array}\right)
$$

The lines in (11) indicates the restrictions to a basis $\left\{e_{i}, i=1,2,3\right\}$ for $\Delta_{2,(1)}^{4}$ for $l=-1$ (by a mild abuse of notation); and the basis $\left\{e_{i}, i=1,2,3,4\right\}$ for $l=0$.

As noted, when the determinant vanishes (e.g. as a polynomial in $\delta$ ) then the indecomposable module $\Delta$ contains a proper submodule $L$. Thus this gives the cases where the algebra is non-semisimple. The roots in the example are given by

$$
\delta= \begin{cases}0, \pm \sqrt{2} & \text { if } l=-1 \\ 0,1, \frac{ \pm \sqrt{17}-1}{2} & \text { if } l=0 \\ 0,0,0,-4,2,2 & \text { if } l=1\end{cases}
$$

The 'classical' first and last cases are known (they may be determined from $[48,55]$ respectively), but the middle case shows that new features appear for intermediate $l$. The details of this example are therefore most intriguing. Here, however, we note only that it will be clear that every such Gram matrix is a finite polynomial in $\delta$. It follows that, with $k=\mathbb{C}$ say, the rank of the form is submaximal only on a Zariski-closed subset of $\delta$-parameter space. In other words: 
Theorem 4.29 For each $l, J_{l, n}(\delta)$ is generically semisimple over $\mathbb{C}$, and non-semisimple in finitely many $\delta$-values.

The example shows that non-semisimple cases exist. But computing Gram determinants is not easy in general. In the classical cases they are most efficiently calculated using translation functors, which combine use of the $F$ and $G$ functors with an alcove-geometric structure obtained via induction and restriction. We begin to address this in Sect. 5 by looking at branching rules.

Remark The semisimplicity condition for the Brauer algebra cases are given explicitly in, for example, $[2,67]$.

\section{Standard Bratteli diagrams}

(5.1) Given a pair of semisimple algebras $A \supset B$, with simple modules labelled by sets $\Lambda(A)$ and $\Lambda(B)$ respectively, then the Bratteli diagram is the graph with vertices $\Lambda(A) \sqcup \Lambda(B)$, and an edge (of multiplicity $m$ ) $\mu \rightarrow v$ whenever simple $B$-module $L_{\mu}$ is a composition factor of the restriction $\operatorname{Res}_{B}^{A} L_{v}$ of simple $A$-module $L_{v}$, of composition multiplicity $m$ (see e.g. [47] for a review and references).

If $A_{0} \supset A_{1} \supset A_{2} \supset \cdots$ is a sequence of subalgebras then the pairwise Bratteli diagrams may be chained together in the obvious way. This is the Bratteli diagram for the sequence.

(5.2) More generally, suppose that $A \supset B$ (or a sequence as above) are quasihereditary algebras, and that the restrictions to $B$ of the standard modules of $A$ have filtrations by the standard modules of $B$ (NB such filtrations, if they exist, have unique multiplicities of factors up to isomorphism, since standard modules are a basis for the Grothendieck group [16]). Then the standard Bratteli diagram encodes the standard filtration multiplicities in the same way as the ordinary Bratteli diagram.

Proposition 5.3 Fix l. There is a standard Bratteli diagram for the tower $\left\{J_{l, n} \subset J_{l, n+1}\right\}_{n \in \mathbb{N}}$ (inclusion by adding a line on the right). The standard restriction rules for the $J_{l, n+1}$-modules $\Delta_{p, \lambda}$ are given as follows.

For $p<l+2$ we have a short exact sequence:

$$
0 \rightarrow \oplus_{i} \Delta_{p-1, \lambda-e_{i}}^{(n)} \rightarrow \operatorname{Res}_{J_{l, n}}^{J_{l, n+1}} \Delta_{p, \lambda}^{(n+1)} \rightarrow \oplus_{i} \Delta_{p+1, \lambda+e_{i}}^{(n)} \rightarrow 0
$$

where the sums are over addable/removable boxes of the Young diagram-hence $\lambda-e_{i}$ denotes $\lambda$ with the $i$-th removable box removed and so on (we have noted the value of $n$ explicitly when different values appear in the same sequence).

For $p=l+2$ :

$$
0 \rightarrow \oplus_{i} \Delta_{p-1, \lambda-e_{i}}^{(n)} \rightarrow \operatorname{Res}_{J_{l, n}}^{J_{l, n+1}} \Delta_{p, \lambda}^{(n+1)} \rightarrow \Delta_{p+1, \lambda}^{(n)} \rightarrow 0
$$

For $p>l+2$ :

$$
0 \rightarrow \Delta_{p-1, \lambda}^{(n)} \rightarrow \operatorname{Res}_{n}^{n+1} \Delta_{p, \lambda}^{(n+1)} \rightarrow \Delta_{p+1, \lambda}^{(n)} \rightarrow 0 .
$$

Proof The proof closely follows [50, Prop.13] or [51]. The main differences are due to the fact that here we are working, for $p>l+2$, with 'inflations' of a subgroup $S_{l+2}$ of $S_{p}$, rather than inflations of $S_{p}$ itself. 
First note that, combinatorially, we may separate the basis $B_{p, \lambda}$ into two subsets: (I) elements in which the component $x$ from $J_{l}^{\|}(n, p, p)$ has the last line propagating (i.e. northern marked-point $n$ is connected to southern marked-point $p^{\prime}$ ); and (II) elements in which it does not (i.e. northern marked-point $n$ is connected to some earlier northern marked-point). For example, in (8) the first three elements have the last line propagating:

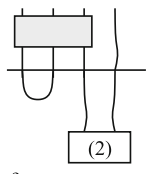

$\mathrm{e}_{1}$

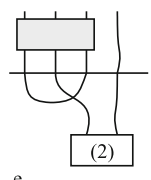

$\mathrm{e}_{4}$

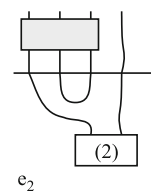

$\mathrm{e}_{2}$

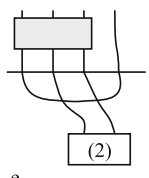

$\mathrm{e}_{6}$

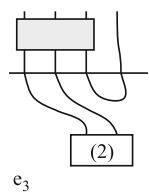

$\mathrm{e}_{3}$

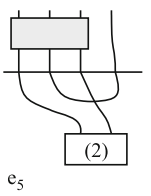

Next note that the subset in (I) is indeed a basis of a submodule with regard to the $J_{l, n-1}$ action (which acts trivially on the last line). For $p>l+2$ it is isomorphic, as it were, to the basis of $\Delta_{p-1, \lambda}^{(n)}$. Furthermore (II) spans a submodule modulo (I)-i.e. it is a basis for the quotient. It is easy to check that this quotient module is $\Delta_{p+1, \lambda}^{(n)}$, noting again that the last line acts trivially, so that there is an isomorphism obtained by 'deforming' the last marked-point from the top to the bottom of the picture (effectively adding another propagating line).

The $p \leq l+2$ cases are similar, except that the deformation takes the 'seed' $S_{|\lambda|}$-module, in the sense of (4.18), from the Specht module $\mathcal{S}_{\lambda}$ to the induction of this module to $S_{|\lambda|+1}$ :
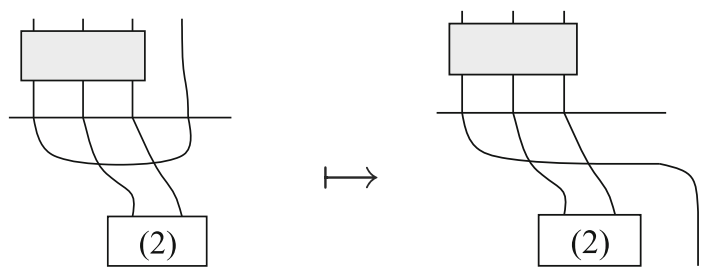

One thus lifts the induction (and in case (I) the restriction) rules for symmetric group Specht modules. Cf. e.g. [50, Prop.13], [25].

(5.4) Note in particular that the restriction is multiplicity free here; and is essentially independent of $n$. The standard Bratteli diagram in the $l=1$ case and in the $l=2$ case is encoded in Fig. 7 , in the form of the corresponding Rollet diagram $\mathrm{R}_{l}$. Rollet diagrams are described, for example, in [47] (and cf. [31]). In brief, a Rollet diagram arises when there is a global (large $n$ ) limit for the index set for standard or simple modules of a tower of algebras (such as $J_{l, n}, n=0,1,2,3, \ldots$ ), induced in the manner of Proposition 4.1; and also for the corresponding restriction rules. Often, as here, there is a global limit for the index sets for odd and even $n$ sequences of algebras separately (localisation and globalisation change $n$ in steps of 2). The Rollet graph is an encoding of all the data in the Bratteli diagram on a vertex set consisting just of the union of the odd and even global limits. One takes this vertex set, and an edge of multiplicity $m$ from $\lambda$ to $\mu$ whenever restriction of $\Delta_{\lambda}$ contains $m$ copies of $\Delta_{\mu}$-this being well-defined by the independence-of- $n$ property.

In particular $\mathrm{R}_{l}$ is an undirected bipartite graph in our case (cf. the directed Bratteli graph). That is, it is multiplicity free, and whenever there is an edge there is an edge in both directions. 

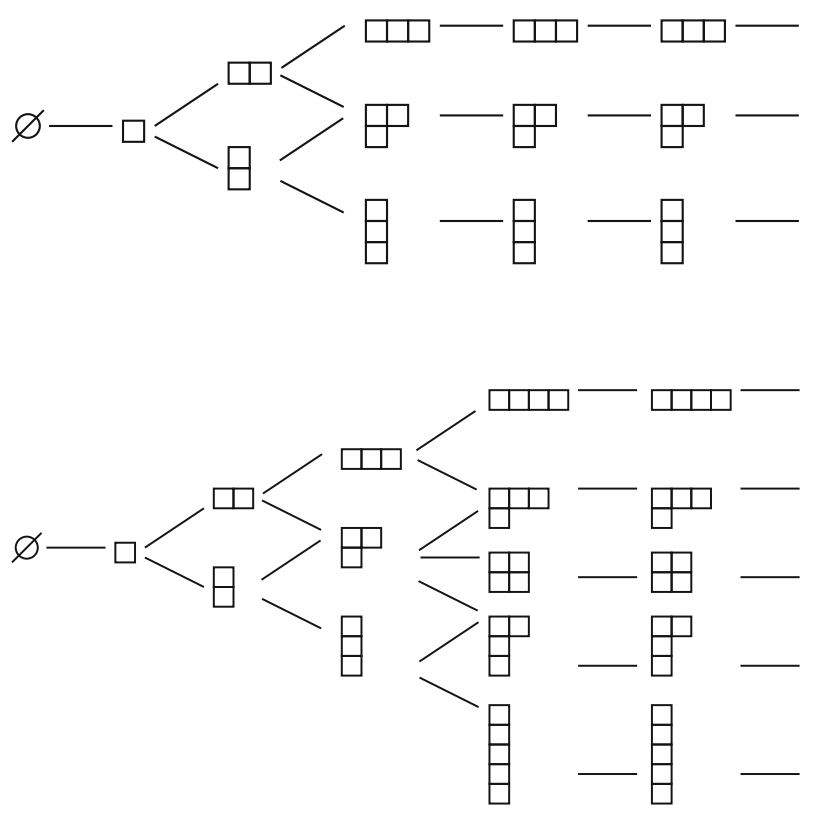

Fig. 7 The $J_{l, n} \subset J_{l, n+1}$ standard Rollet diagram in case $l=1$ and case $l=2$

(5.5) Note that this gives a beautiful combinatorial approach to the dimensions of standard modules, and of the various algebras (cf. $[43,47])$, and in particular the following.

Theorem 5.6 Let $W_{p, \lambda}^{n}(l)$ denote the set of walks of length $n$ on $R_{l}$ from vertex $\emptyset$ to vertex $(p, \lambda)$ (the vertex with label $\lambda$ at distance $p$ from $\emptyset)$. Then $\operatorname{dim}\left(\Delta_{p, \lambda}^{n}\right)=\left|W_{p, \lambda}^{n}(l)\right|$ and $\operatorname{dim}\left(J_{l, n}\right)=\left|W_{0, \emptyset}^{2 n}(l)\right|$.

\section{Discussion}

The construction of $\mathcal{J}_{l}$ and $\mathcal{P}_{l}$ raises many interesting collateral questions. In this section we assemble some brief general observations on our construction, on further developments and on open problems (we defer full details to a separate note [38]).

\subsection{Next steps in reductive representation theory of $J_{I, n}$}

The next steps parallel the program for the Brauer algebra used in [55], but now for each $l$ in turn. Essentially we should compute the blocks, and construct 'translation functors'; and then construct corresponding analogues of Kazhdan-Lusztig polynomials, cf. [19,27,29,55].

We write Ind - for the induction functor adjoint to Res - as in Proposition 5.3 above. The precursor of translation functors in the Brauer case is the natural isomorphism of functors expressed as Ind $\cong \operatorname{Res} G$. The general setup here is as follows.

Lemma 6.1 Suppose we have a sequence of unital algebras $A \supset B \supset C$ and an idempotent $e$ in $A$ such that $e A e \cong C$. Write $\operatorname{Ind}_{C}^{B}: C-\bmod \rightarrow B-\bmod$ for the induction functor corresponding to $C \hookrightarrow B$; similarly for restriction; and $G_{C}^{A}: C-\bmod \rightarrow A-\bmod$ for the 
functor given by $A e \otimes_{C}-\left(c f . G_{e}\right.$ defined as in Proposition 4.26). If $B$ and Ae are isomorphic as left-B right-C-modules then functors $\operatorname{Ind}_{C}^{B} \cong \operatorname{Res}_{B}^{A} G_{C}^{A}$.

Proof We have $\operatorname{Ind}_{C}^{B}-=B_{C} \otimes_{C}-$ and $G_{C}^{A}-=A e \otimes_{C}-$.

In the Brauer case we have the 'disk lemma': Consider a partition in $J(n+1, n+1)$. Replacing the vertex $n+1^{\prime}$ with a vertex $n+2$ defines a map $\eta: J(n+1, n+1) \rightarrow J(n+2, n)$. One easily checks that this is a bijection; and an isomorphism of $n+1, n$-bimodules. On the other hand $B_{n+2} e$ has a basis of partitions in $J(n+2, n+2)$ with a pair $\left\{n+1^{\prime}, n+2^{\prime}\right\}$. There is a natural bijection of this basis with $J(n+2, n)$ (simply omit the indicated pair). Altogether then, $B_{n+1}$ and $B_{n+2} e$ are isomorphic as bimodules. In our case we have the following.

Lemma 6.2 There is a well-defined restriction of $\eta$ to a map $\eta: J_{l, n+1} \rightarrow J_{l, n+2} e$; and this is an isomorphism of left- $J_{l, n+1}$ right- $J_{l, n}$-modules.

Proof One checks that $\eta$ does not change height.

Thus we have the following.

Proposition 6.3 Fix any l. Then for all $n$ we have Ind $\cong \operatorname{Res} G$.

This is a powerful result since, for example, the Ind - functor takes projectives to projectives, while Proposition 5.3 tells us what Res- does to standard modules. Thus we have an iterative scheme for computing the $\Delta$-content of projective modules, cf. [55].

(6.4) Closely related to Proposition 6.3, a generalised Jones Basic Construction [47] applies here (cf. the original Jones Basic Construction [31]). It is analogous to the case in [52].

(6.5) We can use the graphs $R_{l}$ from (5.4) to give an explicit construction for the basis states of the standard modules; and indeed of the entire algebra—a generalised Robinson-Schensted correspondence [41]. See [38] for details.

\subsection{Remarks on $J_{I, n}$ construction}

The $\mathcal{J}_{l}$ construction is amenable to several intriguing generalisations. Here we briefly mention just one particular such generalisation, which case makes a contact with existing studies.

(6.6) The first case with crossings, $J_{l=0, n}$, is connected to the blob algebra [57]:

We say a picture is left-simple if the intersection of the 0 -alcove (as for example in Fig. 4) with the frame of $R$ is connected. A partition is left-simple if every picture of it is left-simple. (For instance the identity in $B_{n}$ is left-simple, while the example in Fig. 4 is not.) Define $J_{\leq l}^{1}(n, m)$ as the subset of $J_{\leq l}(n, m)$ of left-simple partitions.

Remark 6.7 The subspace $k J_{\leq 0}^{1}(n, n)$ is a subalgebra of $J_{0, n}$. This subalgebra is isomorphic to the blob algebra $b_{n-1}\left(q, \bar{q}^{\prime}\right)$, with $q, q^{\prime}$ determined by $\delta$ as follows. Parameterising (as in [57]) with $x=q+q^{-1}$ as the undecorated loop parameter; and $y=q^{\prime}+q^{\prime-1}$ as the decorated loop parameter, we have $x=\delta$ and $y=\frac{\delta+1}{2}$.

Proof (Outline) It is easy to check that the subspace is a subalgebra. It is also easy to show a bijection between $J_{<0}^{1}(n, m)$ and the set of $(n-1, m-1)$-blob diagrams. This does not lift to an algebra map, but shows the dimensions are the same. A heuristic for the algebra 
isomorphism is to note that the intersection of the propagating number zero ideal (4.16) with the subset of left-simple partitions is empty, so there is no $\Delta_{0, \emptyset}$ representation. With this node removed, the Rollet diagram from Sect. 5 becomes a ('doubly-infinite') chain, which is the same as for the blob algebra. See [38] for an explicit proof.

(6.8) On the other hand one can check using Sect. 5 that higher $l$ cases such as the algebra generated by $J_{\leq 1}^{1}(n, n)$ do not coincide with the higher contour algebras [56] or the constructions in $[30,33,70]$.

(6.9) Diagram bases may be used to do graded representation theory (in the general sense, for example, of [13]) for graded blob and TL algebras (see e.g. [63]), regarded as quotients of graded cyclotomic Hecke algebras. It would be interesting to try to generalise [63] to $J_{l, n}$. Of course given an algebra defined via a specific basis as here, there is no reason why the underlying homogeneous basis for a given grading should be the same. There is not a canonical lift of the Plaza-Ryom-Hansen construction to the full Brundan-Kleshchev Hecke algebra [13], and there now exist graded versions of the Brauer algebras $[27,29,45]$ that again do not use the basis of set partitions from this paper. The interpolation $J_{l, n}$ is thus intriguing also from this perspective.

(6.10) As noted (cf. (1.7), (2.16) and (2.19)), $\mathcal{P}, \mathcal{B}, \mathcal{T}$ are monoidal categories with object monoid $(\mathbb{N},+$ ) and monoid composition visualised by lateral (as opposed to vertical) juxtaposition of diagrams. Note however that the categories $\mathcal{J}_{l}, \mathcal{P}_{l}$ do not directly inherit this structure (except in cases $\mathcal{J}_{-1}=\mathcal{T}$ and $\mathcal{J}_{\infty}=\mathcal{B}$ ).

\subsection{Recent developments and further open problems}

There have been several further exciting developments on Brauer algebras since this work was first reported (in arXiv:1401.1774). One should mention again some beautiful new developments on gradings of Brauer algebras, such as the works of Li [45], and Ehring and Stroppel [29]. In particular [29], together with [27], relate Brauer algebras with geometry and alcove geometry. Furthermore both give an answer, for the Brauer algebra, to the problem addressed in (6.9) here.

Also potentially related are Lehrer and Zhang's treatment of duality with orthosymplectic groups [44] and Ehrig and Stroppel's treatment [28] of the Lie superalgebra case (both of which follow on from Benkart et al. [5]). The restriction of this 'super' construction to the corresponding TL algebra has been considered [53], but the general $l$ case is open.

For TL there is a module defined over a suitable integral ground ring that base-changes to a full-tilting module in every quasihereditary specialisation. Indeed in this case the Schur-Weyl duality provides such a module $[24,49,66]$. It would be interesting to have such a uniform construction for full-tilting modules for all the Brauer algebras, so the $J_{l, n}$ interpolation is interesting from this perspective.

Related to the last point, it is very interesting to ask if there is a lift of our construction to generalisations such as the Birman-Murakami-Wenzl (BMW) algebra [8,40,61,62,72]. See also e.g. $[1,3,65]$.

Acknowledgements PM would like to thank Robert Marsh for useful conversations. ZK and PM would like to thank EPSRC for financial support (EP/I038683/1).

Open Access This article is distributed under the terms of the Creative Commons Attribution 4.0 International License (http://creativecommons.org/licenses/by/4.0/), which permits unrestricted use, distribution, and repro- 
duction in any medium, provided you give appropriate credit to the original author(s) and the source, provide a link to the Creative Commons license, and indicate if changes were made.

\section{References}

1. Alvarez, M., Martin, P.P.: A Temperley-Lieb category for 2-manifolds. Preprint (2006). arXiv:0711.4777

2. Andersen, H.H., Stroppel, C., Tubbenhauer, D.: Semisimplicity of Hecke and (walled) Brauer algebras. J. Aust. Math. Soc. 103(1), 1-44 (2017). https://doi.org/10.1017/S1446788716000392

3. Baez, J., Dolan, J.: Higher-dimensional algebra and topological quantum field theory. J. Math. Phys. 36, 6073-6105 (1995). arXiv:q-alg/9503002

4. Baxter, R.J.: Exactly Solved Models in Statistical Mechanics. Academic Press, New York (1982)

5. Benkart, G., Shader, C.L., Ram, A.: Tensor product representations for orthosymplectic Lie superalgebras. J. Pure Appl. Algebra 130, 1-48 (1998)

6. Benson, D.J.: Representations and Cohomology I. Cambridge University Press, Cambridge (1995)

7. Bethe, H.: Zur Theorie der Metalle. Z. Phys. 71, 205-226 (1931)

8. Birman, J.S., Wenzl, H.: Braids, link polynomials and a new algebra. Trans. AMS 313, 249-273 (1989)

9. Bjorken, J.D., Drell, S.D.: Relativistic Quantum Fields. McGraw-Hill, New York (1965)

10. Brauer, R.: On algebras which are connected with the semi-simple continuous groups. Ann. Math. 38, 854-872 (1937)

11. Brauer, R.: On modular and p-adic representations of algebras. Proc. Natl. Acad. Sci. USA 25, 252-258 (1939)

12. Brown, W.P.: An algebra related to the orthogonal group. Mich. Math. J. 3, 1-22 (1955-1956)

13. Brundan, J., Kleshchev, A.: Blocks of cyclotomic Hecke algebras and Khovanov-Lauda algebras. Invent. Math. 178, 451-484 (2009)

14. Candu, C., Saleur, H.: A lattice approach to the conformal $O S p(2 s+2 \mid 2 s)$ supercoset sigma model. Part I: Algebraic structures in the spin chain. The Brauer algebra. Nucl. Phys. B 808, 441-486 (2009)

15. Cautis, S., Jackson, D.M.: On Tutte's chromatic invariant. Trans. AMS 362, 509-535 (2010)

16. Cline, E., Parshall, B., Scott, L.: Finite-dimensional algebras and highest weight categories. J. Reine Angew. Math. 391, 85-99 (1988)

17. Cline, E., Parshall, B., Scott, L.: Generic and $q$-rational representation theory. Publ. RIMS 35, 31-90 (1999)

18. Cox, A.G., Martin, P.P., Parker, A.E., Xi, C.C.: Representation theory of towers of recollement: theory, notes and examples. J. Algebra 302, 340-360 (2006). arXiv:math.RT/0411395

19. Cox, A.G., De Visscher, M., Martin, P.P.: A geometric characterisation of the blocks of the Brauer algebra. JLMS 80, 471-494 (2009). arXiv:math.RT/0612584

20. Crowell, R.H., Fox, R.H.: Introduction to Knot Theory. Ginn, Boston, New York (1963)

21. Dlab, V., Ringel, C.M.: A construction for quasi-hereditary algebras. Compos. Math. 70, 155-175 (1989)

22. Dlab, V., Ringel, C.M.: Quasi-hereditary algebras. Ill. J. Math. 33, 280-291 (1989)

23. Doikou, A., Martin, P.P.: Hecke algebraic approach to the reflection equation for spin chains. J. Phys. A 36, 2203-2225 (2003). arXiv:hep-th/0206076

24. Donkin, S.: The $q$-Schur Algebra. LMS Lecture Notes Series, vol. 253. Cambridge University Press, Cambridge (1998)

25. Doran, W.F., Wales, D.B., Hanlon, P.J.: On semisimplicity of the Brauer centralizer algebras. J. Algebra 211, 647-685 (1999)

26. Du, J., Parshall, B., Scott, L.: Quantum Weyl reciprocity and tilting modules. Commun. Math. Phys. 195, 321-352 (1998)

27. Ehrig, M., Stroppel, C.: Nazarov-Wenzl algebras, coideal subalgebras and categorified skew Howe duality. Adv. Math. 331, 58-142 (2018). https://doi.org/10.1016/j.aim.2018.01.013

28. Ehrig, M., Stroppel, C.: Schur-Weyl duality for the Brauer algebra and the ortho-symplectic Lie superalgebra. Mathematische Zeitschrift 284(1-2), 595-613 (2016)

29. Ehrig, M., Stroppel, C.: Koszul gradings on Brauer algebras. Int. Math. Res. Not. 2016(13), 3970-4011 (2016). https://doi.org/10.1093/imrn/rnv267

30. Fan, C.K., Green, R.M.: On the affine Temperley-Lieb algebras. J. LMS 60, 366-380 (1999)

31. Goodman, F.M., de la Harpe, P., Jones, V.F.R.: Coxeter-Dynkin Diagrams and Towers of Algebras. Mathematical Sciences Research Institute Publication, Springer, Berlin (1989)

32. Green, J.A.: Polynomial Representations of $G L_{n}$. Springer, Berlin (1980)

33. Green, R.M.: Generalized Temperley-Lieb algebras and decorated tangles. J. Knot Theory Ramif. 7, 155-171 (1998) 
34. Hanlon, P., Wales, D.: A tower construction for the radical in Brauer's centralizer algebras. J. Algebra 164, 773-830 (1994)

35. James, G.D.: The Representation Theory of the Symmetric Groups. Lecture Notes in Mathematics, vol. 682. Springer, Berlin (1978)

36. James, G.D., Kerber, A.: The Representation Theory of the Symmetric Group. Addison-Wesley, London (1981)

37. Jantzen, J.C.: Representations of Algebraic Groups. Academic Press, New York (1987)

38. Kadar, Z., Martin, P.: Geometric partition categories (2014) (in preparation)

39. Kassel, C.: Quantum Groups. Springer, Berlin (1995)

40. Kauffman, L.H.: Knots and Physics. World Scientific, Singapore (1991)

41. Knuth, D.E.: Sorting and Searching. The Art of Computer Programming, vol. 3, 2nd edn. Addison Wesley, Boston (1998)

42. Koenig, S., Xi, C.C.: Cellular algebras: inflations and Morita equivalences. J. LMS 60, 700-722 (1999)

43. Leduc, R., Ram, A.: A ribbon Hopf algebra approach to the irreducible representations of centralizer algebras: the Brauer, Birman-Wenzl, and type- $A$ Iwahori-Hecke algebras. Adv. Math. 125, 1-94 (1997)

44. Lehrer, G., Zhang, R.: The first fundamental theorem of invariant theory for the orthosymplectic supergroup. Commun. Math. Phys. 349(2), 661-702 (2017)

45. Li, G.: A KLR Grading of the Brauer Algebras. arXiv:1409.1195

46. Lieb, E.H., Mattis, D.C.: Mathematical Physics in One Dimension. Academic Press, New York (1966)

47. Marsh, R., Martin, P.: Pascal arrays: counting Catalan sets. Preprint (2006). arXiv:math/0612572v1 [math.CO]

48. Martin, P.: Potts Models and Related Problems in Statistical Mechanics. World Scientific, Singapore (1991)

49. Martin, P.P.: On Schur-Weyl duality, $A_{n}$ Hecke algebras and quantum $s l(N)$. Int. J. Mod. Phys. A 7(suppl.1B), 645-674 (1992)

50. Martin, P.P.: Temperley-Lieb algebras for non-planar statistical mechanics- the partition algebra construction. J. Knot Theory Ramif. 3(1), 51-82 (1994)

51. Martin, P.P.: The structure of the partition algebras. J. Algebra 183, 319-358 (1996)

52. Martin, P.P.: The partition algebra and the Potts model transfer matrix spectrum in high dimensions. J. Phys. A 32, 3669-3695 (2000)

53. Martin, P.P.: On diagram categories, representation theory and statistical mechanics. AMS Contemp. Math. 456, 99-136 (2008)

54. Martin, P.P.: Lecture Notes in Representation Theory. Unpublished Lecture Notes (2009)

55. Martin, P.P.: The decomposition matrices of the Brauer algebra over the complex field. Trans. AMS 367, 1797-1825 (2015). arXiv:0908.1500 (2009)

56. Martin, P.P., Green, R.M., Parker, A.E.: Towers of recollement and bases for diagram algebras: planar diagrams and a little beyond. J. Algebra 316, 392-452 (2007). arXiv:math.RT/0610971

57. Martin, P.P., Saleur, H.: The blob algebra and the periodic Temperley-Lieb algebra. Lett. Math. Phys. 30, 189-206 (1994). arXiv:hep-th/9302094

58. Martin, P.P., Woodcock, D.: On the structure of the blob algebra. J. Algebra 225, 957-988 (2000)

59. Mazorchuk, V.: On the structure of Brauer semigroup and its partial analogue. Probl. Algebra 13, 29-45 (1998)

60. Moise, E.E.: Geometric Topology in Dimensions 2 and 3. Graduate Texts in Mathematics, vol. 47. Springer, New York (1977)

61. Morton, H.R.: A basis for the Birman-Murakami-Wenzl algebra. Unpublished (2010)

62. Murakami, J.: The Kauffman polynomial of links and representation theory. Osaka J. Math. 24(4), 745758 (1987)

63. Plaza, D., Ryom-Hansen, S.: Graded cellular bases for Temperley-Lieb algebras of type A and B. J. Algebraic Comb. 40(1), 137-177 (2014)

64. Putcha, M.: Complex representations of finite monoids II. Highest weight categories and quivers. J. Algebra 205, 53-76 (1998)

65. Reshetikhin, N.Y., Turaev, V.G.: Ribbon graphs and their invariants derived from quantum groups. Commun. Math. Phys. 127, 1-26 (1990)

66. Ringel, C.M.: The category of modules with good filtrations over a quasi-hereditary algebra has almost split sequences. Math. Z. 208, 209-225 (1991)

67. Rui, H.: A criterion on the semisimple Brauer algebras. J. Comb. Theory Ser. A 111, $78-88$ (2005)

68. Serganova, V.: Characters of irreducible representations of simple Lie superalgebras. Doc. Math. 583-593 (1998) 
69. Temperley, H.N.V., Lieb, E.H.: Relations between percolation and colouring problems and other graph theoretical problems associated with regular planar lattices: some exact results for the percolation problem. Proc. R. Soc. A 322, 251-280 (1971)

70. tom Dieck, T.: Categories of rooted cylinder ribbons and their representations. J. Reine Angew. Math. 494, 35-63 (1998)

71. Wenzl, H.: On the structure of Brauer's centralizer algebras. Ann. Math. 128, 173-193 (1988)

72. Yu, S.: The cyclotomic Birman-Murakami-Wenzl algebras. Ph.D. thesis, The University of Sydney (2007). arXiv:0810.0069

Publisher's Note Springer Nature remains neutral with regard to jurisdictional claims in published maps and institutional affiliations. 